\title{
ACUPUNCTURE DANS LA PRÉVENTION, LE TRAITEMENT DES BLESSURES ET L'AMÉLIORATION DE LA PERFORMANCE CHEZ LES ATHLÈTES: REVUE DE LITTÉRATURE
}

\section{ARTICLE D'EXAMEN}

JUNIOR, Julio Cesar Dias ${ }^{1}$

Junior, Julio Cesar Dias. Acupuncture dans la prévention, le traitement des blessures et l'amélioration de la performance chez les athlètes: Revue de littérature. Revista Científica Multidisciplinar Núcleo do Conhecimento. 04 année, Ed. 10, vol. 10, pp. 59-98. octobre 2019. ISSN: 2448-0959, Lien d'accès: https://www.nucleodoconhecimento.com.br/sante/acupuncture-enprevention

\section{RÉSUMÉ}

Des études ont montré que l'acupuncture améliore la performance physique chez les athlètes professionnels, mais le gros problème est les blessures sportives, qui peuvent

${ }^{1}$ Diplôme en physiothérapie du Centre Universitaire d'Araraquara - UNIARA - 2005 (Araraquara-SP); Lato sensu études supérieures en orthopédie et physiothérapie traumatologique de l'Institut Cohen - Orthopédie, réadaptation et médecine du sport 2006 (Sao Paulo-SP)Formation en thérapie manuelle ostéopathique par I'Institut Cefisa - 2008 (Araraquara-SP); Amélioration professionnelle dans la rééducation posturale mondiale et la reprogrammation des moteurs sensoriels - RPG/RSM par le Paulista Institute of Systemic Studies - IPES - 2010 (Ribeiro Preto-SP); Études supérieures Lato sensu en acupuncture systémique de I'Institut Paulista d'études systémiques - IPES - 2013 (Ribeiro Preto-SP); Stricto sensu Maîtrise postdoctorale en développement territorial et environnement du Centre Universitaire d'Araraquara UNIARA - 2016 (Araraquara - SP); Amélioration professionnelle du Pilates Clinique et Fonctionnel par l'Institut Paulista d'Études Systémiques - IPES - 2016 (Ribeiro Preto$\mathrm{SP})$. 
les laisser loin des activités pendant de longues périodes. L'étude a mené une revue de littérature, recherchant l'efficacité de la technique dans la prévention, l'amélioration de la performance et dans le traitement des lésions. Certains auteurs illustrent et clarifient le processus de neurophysiologie de la douleur et la façon dont l'acupuncture peut intervenir dans ce problème, d'autres, développent et appliquent des protocoles pour obtenir une amélioration des performances, le traitement des maladies et la prévention des Blessure. Parmi les résultats ont été trouvés quatre-vingt-neuf articles sur le sujet, où: vingt-deux ont signalé le traitement des lésions et trente-sept la prévention et l'amélioration de la performance athlétique, la direction de la demande une fois dans la semaine, de 20 - 30 minutes, présentant résultats satisfaisants pour les données analysées. Sur la base de cette littérature et de la pratique fondée sur des preuves, on peut conclure que l'acupuncture est une excellente intervention pour atteindre ces objectifs dans le domaine du sport.

Mots-clés: Acupuncture, blessures, douleur, performance.

\section{INTRODUCTION}

Depuis plusieurs millénaires, l'acupuncture est utilisée comme une forme de prévention et de traitement de diverses maladies du corps. Cette technique de la médecine chinoise a manipulé des aiguilles de pierres et de boutons de poisson tout au long de l'âge de pierre (3000 ans avant JC). ${ }^{1,2}$ L'empereur jaune classique : Huang Di Nei Jing, a constitué l'un des plus anciens enregistrements de la médecine chinoise traditionnelle (MTC), intégrant à nos jours les bases philosophiques, qui conceptualise l'équilibre de l'univers et de la nature, que dans sa vision (chinoise), l'énergie s'appelle Chi ou Qi. Ce facteur a deux principes antagonistes qui sont achevés et ont tendance à être en équilibre, même si elle est sous l'influence de l'univers, appelé Yin et Yang.

L'acupuncture est définie comme l'harmonisation de l'individu avec l'environnement dans lequel il est, non seulement, d'insérer des aiguilles et de remédier au problème de la maladie, mais de réaliser l'idéal de prévention de diverses pathologies. ${ }^{4,5} \mathrm{C}$ 'est une procédure effectuée avec objectif thérapeutique, n'est pas toxique, sans 
I'utilisation d'éléments chimiques, avec des effets secondaires minimes, bien qu'il soit capable de libérer des substances endogènes telles que les neurotransmetteurs. ${ }^{4,6}$, $7,8,9$

L'intervention est de stimuler les acupoints pour atteindre l'effet thérapeutique désiré et atteindre l'homéostasie de l'organisme. De nombreuses études illustrent les effets de la théorie neuronale non opioïde (inhibe les impulsions nerveuses agissant sur le système nerveux périphérique et central); théorie de l'humour (stimule la libération d'opioïdes endogènes, d'autres hormones et neurotransmetteurs). 10, 11 But dans le contrôle de la douleur, l'association des aiguilles avec le courant électrique, appelé électroacupuncture, potentialiates et présente des avantages dans le pratique clinique, en plus d'être utilisé aussi comme: l'acupuncture auriculaire; crindement; moxabusto; ventouses d'aspiration; l'acupuncture au laser. 3, 10

Dans le sport, l'utilisation de la technologie à la recherche de meilleurs résultats continue de croître, dépassant les limites du corps humain. Plusieurs thérapies naturelles ou intégratives sont étudiées comme source de thérapie complémentaire pour améliorer les conditions sportives. Bien que le nombre d'études soit rare, l'acceptation de l'acupuncture est encore très curieuse, car de nombreuses personnes doutent encore des preuves scientifiques. ${ }^{9}$ Cependant, son utilisation dans l'environnement sportif a augmenté, agissant dans le traitement de la douleur et des blessures athlétiques, accélérant le processus de rétablissement de l'individu. Une autre question présentée est l'intervention efficace dans l'amélioration de la performance physique et la prévention des blessures causées par les athlètes amateurs et professionnels 12, 13, 14 .

\section{BUT}

L'objectif de ce travail était de mener une étude bibliographique de l'utilisation de l'acupuncture dans la prévention, l'amélioration de la performance athlétique et dans le traitement des blessures dans diverses modalités sportives. 


\section{MÉTHODOLOGIE}

Une enquête bibliographique a été menée dans les bases de données Scielo, Google Acadêmico et Scoopus, afin d'obtenir des informations sur : l'acupuncture dans la

prévention, l'amélioration des performances et le traitement des blessures sportives. Les mots clés suivants ont été utilisés : acupuncture; l'acupuncture et la douleur; l'acupuncture dans la prévention; les blessures sportives et l'acupuncture; l'acupuncture et l'amélioration de la performance athlétique; traitement des blessures et de l'acupuncture.

Pour délimiter la découpe de cette étude, un premier dépistage a été effectué parmi les nombreux articles trouvés, totalisant 89 manuscrits, sélectionnant 22 qui traitaient du sujet d'acupuncture dans le traitement des blessures et 37 portant sur la prévention des blessures sportives et l'amélioration des performances athlétiques. Sur la base de cette analyse, il convient de mentionner que de toutes les études trouvées principalement, l'univers de la recherche comprenait 59 articles publiés dans des revues scientifiques, des domaines de congrès, des livres, des sites Web, qui dans le titre, abstrait ou mots-clés terminologies utilisées pendant la recherche.

\section{RESULTATS ET DISCUSSION}

Dans cette recherche a abouti à plusieurs articles (graphique 1; tableau 1), ayant été exécuté isolément par les mots clés.

Graphique 1 : Nombre total d'articles trouvés et utilisés dans la recherche.

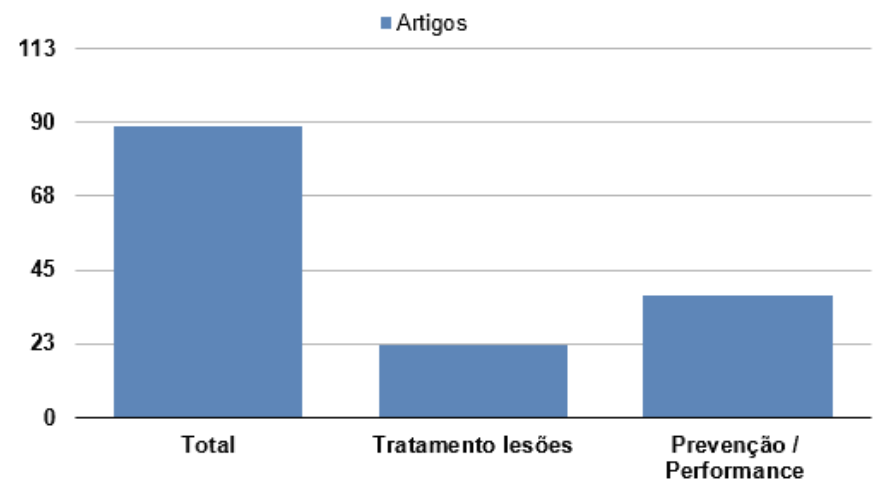


Tableau 1 : Articles relatifs à la prévention, à l'amélioration de la performance athlétique et au traitement des blessures sportives.

\begin{tabular}{|c|c|c|}
\hline Auteurs & Titre de l'article & Journals/Anais/Congresses/Sites \\
\hline $\begin{array}{l}\text { Ehrlich D, Haber } \\
\text { P, } 1992\end{array}$ & $\begin{array}{l}\text { Influence of } \\
\text { acupuncture on pysical } \\
\text { performance capacity } \\
\text { and Haemodynamic } \\
\text { Parameters }\end{array}$ & J. Sports Med \\
\hline $\begin{array}{lr}\text { Santos } & \text { VC, } \\
\text { Kawano } & \text { MM, } \\
\text { Banja RA, } 2008\end{array}$ & $\begin{array}{l}\text { Acupuntura na melhora } \\
\text { da performance em } \\
\text { atletas juvenis de } \\
\text { handebol }\end{array}$ & Rev Saúde e Pesq \\
\hline $\begin{array}{l}\text { Rubio K, Godoy } \\
\text { Moreira F, } 2008\end{array}$ & $\begin{array}{l}\text { A dor em corredores } \\
\text { com fascite plantar: o } \\
\text { uso da acupuntura }\end{array}$ & Rev Dor \\
\hline $\begin{array}{ll}\text { Pires } & \text { TF, } \\
\text { Pellegrinotti } & \text { IL, } \\
2010 & \end{array}$ & $\begin{array}{l}\text { Acupuntura na } \\
\text { Performance Atlética: } \\
\text { Estudo Exploratório }\end{array}$ & 8 Mostra Acadêmica da UNIMEP \\
\hline $\begin{array}{l}\text { Yang HY, Liu TY, } \\
\text { Gao M, } 2006\end{array}$ & $\begin{array}{l}\text { Electrical acupoint } \\
\text { stimulation increases } \\
\text { athletes rapid strength }\end{array}$ & Zhongguo Zhen Jiu \\
\hline $\begin{array}{ll}\text { Luna } & \text { MP, } \\
\text { Fernandes } & \text { Filho } \\
\text { J, } 2005 & \end{array}$ & $\begin{array}{l}\text { Efeitos da Acupuntura } \\
\text { na performance de } \\
\text { Atletas velocista de alto } \\
\text { rendimento do Rio de } \\
\text { Janeiro }\end{array}$ & Fit e Perform J \\
\hline $\begin{array}{l}\text { Dias Junior JC, } \\
\text { Marino DM, } 2019\end{array}$ & $\begin{array}{l}\text { Acupuntura na } \\
\text { prevenção de lesões } \\
\text { musculares em atletas } \\
\text { de futebol profissional }\end{array}$ & Rev Fisiot S Fun \\
\hline
\end{tabular}




\begin{tabular}{|c|c|c|}
\hline Maciocia G, 2007 & $\begin{array}{l}\text { Os fundamentos da } \\
\text { medicina chinesa: um } \\
\text { texto abrangente para } \\
\text { acupunturistas } \\
\text { fitoterapeutas }\end{array}$ & Livre \\
\hline $\begin{array}{ll}\text { Akimoto } & \text { T, } \\
\text { Nakahori } & \text { C, } \\
\text { Aizawa } & \text { K, } \\
\text { Kimura } & \text { F, } \\
\text { Fukubayashi } & \text { T, } \\
\text { Kono I, 2003 } & \end{array}$ & $\begin{array}{l}\text { Acupuncture and } \\
\text { responses of imunoligic } \\
\text { and endocrine markers } \\
\text { during competition }\end{array}$ & Med Sci in Sports Exerc \\
\hline $\begin{array}{ll}\text { Karvelas } & \text { BR, } \\
\text { Hoffman } & \text { MD, } \\
\text { Zeni Al, } 1996\end{array}$ & $\begin{array}{l}\text { Acute Effects of } \\
\text { Acupuncture on } \\
\text { Physiological and } \\
\text { Psychological } \\
\text { Responses to Cycle } \\
\text { Ergometry }\end{array}$ & Arch Phys Med Rehabil \\
\hline $\begin{array}{l}\text { Fry AC, Kraemer } \\
\text { KJ, } 1997\end{array}$ & $\begin{array}{l}\text { Resistance exercise ov } \\
\text { ertraing } \\
\text { and overreaching: neur } \\
\text { oendocrine responses }\end{array}$ & Sports Med \\
\hline KnardahI S, 1998 & $\begin{array}{l}\text { Sympathetic } \\
\text { nerve activity after } \\
\text { acupuncture in humans }\end{array}$ & $\begin{array}{l}\text { Department of } \\
\text { Neurophysiology }\end{array}$ \\
\hline $\begin{array}{l}\text { Barlas PJ, } \\
\text { Robinson JA, Ba } \\
\text { xter GD, } 2000\end{array}$ & $\begin{array}{l}\text { Lack ofeffect of acupun } \\
\text { cture upon signs } \\
\text { and symptoms of delay } \\
\text { ed } \\
\text { onset muscle soreness }\end{array}$ & Clinical Physio \\
\hline $\begin{array}{l}\text { Rossetto SC, } \\
2009\end{array}$ & $\begin{array}{l}\text { Acupuntura nos } \\
\text { Esportes }\end{array}$ & Livre \\
\hline
\end{tabular}




\begin{tabular}{|c|c|c|}
\hline $\begin{array}{l}\text { França D, } \\
\text { Fernandes- } \\
\text { Senna V, Cortez } \\
\text { CM, } 2004\end{array}$ & 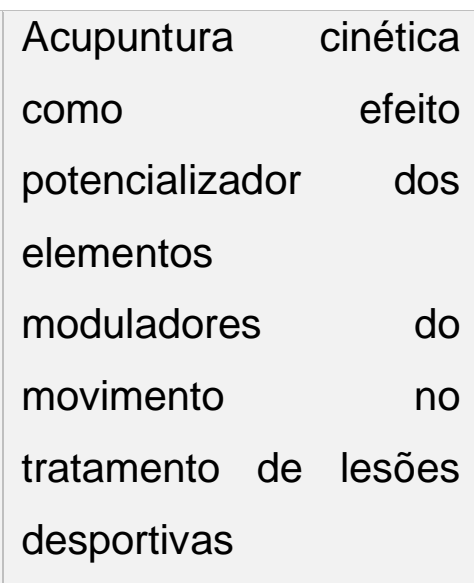 & Fisioter Bras \\
\hline $\begin{array}{l}\text { Wadsworth L T, } \\
2006\end{array}$ & $\begin{array}{l}\text { Acupuncture in sports } \\
\text { medicine }\end{array}$ & Curr Sports Med Rep \\
\hline Barela J A, 2000 & $\begin{array}{l}\text { Estratégias de Controle } \\
\text { em Movimentos } \\
\text { Complexos: Ciclo } \\
\text { Percepção - Ação no } \\
\text { Controle Postural }\end{array}$ & Rev Paul de Educ Fís \\
\hline $\begin{array}{l}\text { Papler PG et al., } \\
1999\end{array}$ & $\begin{array}{l}\text { Reabilitação do joelho. } \\
\text { In: Greve J.M. A. e } \\
\text { Amattuzzi, } \\
\text { Medicina M.M. } \\
\text { reabilitação aplicada à } \\
\text { ortopedia } \\
\text { traumatologia }\end{array}$ & Livre \\
\hline $\begin{array}{l}\text { Gemeo LH. } \\
\text { Ignatti C, } 2004\end{array}$ & $\begin{array}{l}\text { Acupuntura como } \\
\text { Ferramenta Auxiliar do } \\
\text { Aumento da } \\
\text { Performance } \\
\text { Desportiva. In: Anais do } \\
\text { Simpósio Internacional } \\
\text { de Ciências Integradas } \\
\text { da UNAERP }\end{array}$ & Anais Simpósio \\
\hline
\end{tabular}




\begin{tabular}{|c|c|c|}
\hline Frasca L, 2011 & $\begin{array}{l}\text { Desempenho na Ponta } \\
\text { da Agulha }\end{array}$ & Rev Farmac \\
\hline Costa V, 2013 & $\begin{array}{lr}\text { Acupuntura } & \text { Previne } \\
\text { Lesões e ajuda a } & \\
\text { melhorar } & \text { o } \\
\text { Desempenho } & \text { nas } \\
\text { Pistas [periódico na } \\
\text { internet] }\end{array}$ & $\begin{array}{l}\text { http://blogs.oglobo.globo.com/pulso/ } \\
\text { post/acupuntura-previne-lesoes- } \\
\text { ajuda-melhorar-desempenho-nas- } \\
\text { pistas-500798.html. }\end{array}$ \\
\hline Luna M, 2016 & $\begin{array}{l}\text { Os Benefícios da } \\
\text { Acupuntura no Esporte }\end{array}$ & $\begin{array}{l}\text { http://www.ibramrp.com.br/noticia/49 } \\
\text { /os-beneficios-da-acupuntura-no- } \\
\text { esporte }\end{array}$ \\
\hline $\begin{array}{l}\text { Bopp-Limoge C, } \\
1998\end{array}$ & $\begin{array}{l}\text { L’acupuncture Permet- } \\
\text { elle D`ameliorer les } \\
\text { Performances } \\
\text { Sportives Stude } \\
\text { Personnelle a Propos } \\
\text { de } 35 \text { Sportifs de haut } \\
\text { niveau. }\end{array}$ & These Medicine \\
\hline Lin ZP et al., 2009 & $\begin{array}{l}\text { Effects of acupuncture } \\
\text { stimulation on recovery } \\
\text { ability for male elite } \\
\text { basketball athletes }\end{array}$ & Amer Jour of Chin Med \\
\hline $\begin{array}{l}\text { Lin, ZP et al., } \\
2009\end{array}$ & $\begin{array}{l}\text { Effect of auricular } \\
\text { acupuncture on oxygen } \\
\text { consumption of boxing } \\
\text { athletes }\end{array}$ & Chin Med Jour \\
\hline $\begin{array}{l}\text { Dhillon S et al., } \\
2008\end{array}$ & $\begin{array}{l}\text { The acute effect of } \\
\text { acupuncture on } 20-\mathrm{km} \\
\text { cycling performance. }\end{array}$ & Clin Jour of Spo Med \\
\hline $\begin{array}{l}\text { Hubscher M et } \\
\text { al., } 2010\end{array}$ & $\begin{array}{l}\text { Immediate effects of } \\
\text { acupuncture }\end{array}$ & Euro Jour of Appli Physi \\
\hline
\end{tabular}




\begin{tabular}{|c|c|c|}
\hline & $\begin{array}{l}\text { strength performance: a } \\
\text { ramdomized, controlled } \\
\text { crossover trial }\end{array}$ & \\
\hline $\begin{array}{l}\text { Geng } L \text { J et al., } \\
1995\end{array}$ & $\begin{array}{l}\text { Investigation on the } \\
\text { effects of ear } \\
\text { acupressure on } \\
\text { exercise-induced lactic } \\
\text { acid levels and the } \\
\text { implications for athletic } \\
\text { training }\end{array}$ & Amer Jour of Acupu \\
\hline Franco RS, 2012 & $\begin{array}{l}\text { Avaliação do Efeito da } \\
\text { Acupuntura sobre o } \\
\text { Desempenho Físico } \\
\text { pelo Teste do Banco de } \\
\text { Harvard }\end{array}$ & Dissertação \\
\hline $\begin{array}{l}\text { Fonseca LP, } \\
\text { Lessa JFM, } 2011\end{array}$ & $\begin{array}{l}\text { Efeito da Aplicação da } \\
\text { Acupuntura na } \\
\text { Resistência Muscular } \\
\text { Localizada de Membros } \\
\text { Superiores em } \\
\text { Praticantes de } \\
\text { Exercício Resistido. }\end{array}$ & Mobografia \\
\hline $\begin{array}{lr}\text { Belmiro } & \text { H, } \\
\text { Vicentini } & \text { D, } \\
\text { Camilotti } & \text { CM, } \\
2013 & \end{array}$ & $\begin{array}{l}\text { Efeitos da Acupuntura } \\
\text { no Desempenho Motor } \\
\text { de Atletas. }\end{array}$ & Rev Fac Educ Fis \\
\hline $\begin{array}{l}\text { Rubio K; Godoy } \\
\text { Moreira F, } 2007\end{array}$ & $\begin{array}{l}\text { A representação da dor } \\
\text { em atletas olímpicos } \\
\text { brasileiros }\end{array}$ & Rev Dor \\
\hline Parisotto D, 2014 & $\begin{array}{ll}\text { Efeito Imediato } & \mathrm{da} \\
\text { Aplicação } & \mathrm{da}\end{array}$ & Dissertação \\
\hline
\end{tabular}




\begin{tabular}{|c|c|c|}
\hline & $\begin{array}{l}\text { Acupuntura na Dor } \\
\text { Muscular Tardia e na } \\
\text { Capacidade de } \\
\text { Contração Muscular }\end{array}$ & \\
\hline Staud R, 2007 & $\begin{array}{l}\text { Mechanisms of } \\
\text { acupunture analgesia: } \\
\text { effective therapy for } \\
\text { musculoskeletal pain? }\end{array}$ & Curr rheumatol Rep \\
\hline $\begin{array}{l}\text { Bucinskaite V, } \\
\text { Lundeberg T, } \\
\text { Stenfors C, } \\
\text { Ekblom A, Dahlin } \\
\text { L, Theodorsson } \\
\text { E, } 1994\end{array}$ & $\begin{array}{l}\text { Effects of electro- } \\
\text { acupuncture and } \\
\text { pysical exercice on } \\
\text { regional concentrations } \\
\text { of neuropeptides in rat } \\
\text { brain }\end{array}$ & Brain research \\
\hline Shang C, 2009 & $\begin{array}{l}\text { Prospective tests on } \\
\text { biological models of } \\
\text { acupuncture }\end{array}$ & $\begin{array}{l}\text { Evid Based Complement Alternat } \\
\text { Med. }\end{array}$ \\
\hline Bowsher D,1988 & $\begin{array}{l}\text { Mechanisms of } \\
\text { acupuncture. In: Filshie } \\
\mathrm{J} \text {, White A. editors. } \\
\text { Medial Acupuncture: a } \\
\text { western scientific } \\
\text { approach. }\end{array}$ & Livre \\
\hline Ma SX, 2004 & $\begin{array}{l}\text { Neurobiology } \\
\text { acupuncture: } \\
\text { CAM }\end{array}$ & $\begin{array}{l}\text { Evid Based Complement Alternat } \\
\text { Med }\end{array}$ \\
\hline $\begin{array}{l}\text { Li J, Wang Q, } \\
\text { Liang H, Dong H, } \\
\text { Li Y, Ng EH, et al., } \\
2012\end{array}$ & $\begin{array}{l}\text { Biophysical } \\
\text { characteristics of } \\
\text { meridians and } \\
\text { acupoints: a systematic } \\
\text { review. }\end{array}$ & $\begin{array}{l}\text { Evid Based Complement Alternat } \\
\text { Med. }\end{array}$ \\
\hline
\end{tabular}


Lee, SH, Chung, Effects Acupunturaq on Neurocienses Letters

$\mathrm{SH}$, Lee, JS, Kim, the 5-

SS, Shin, HD, hydroxytryptamine

Lim, BV, et al., synthesis and

2002 tryptophan hydroxylase

expression in dorsal in

the dorsal raphe of

exercice rats.

Zyloney CE, Imaging the functional Mol pain

Jensen K, Polich connectivity of the

G, Loiotile RE, Periaqueductal Gray

Cheetham A, during genuine and

LaViolette PS, et sham

al., 2010 electroacupuncture treatment.

Minori AET, Mejia Atuação da Acupuntura Monografia

DPM, 2007 para o Tratamento de LER/DORT no Ombro.

$\begin{array}{lrlr}\text { Brum KN, } & \text { Tratamento } & \text { de } \\ \text { Alonso, AC, } & \text { massagem } & \text { e } \\ \text { Brech GC, 2009 } & \text { acupuntura } & \text { em } \\ & \text { corredoresrecreacionai } \\ & \text { s com síndrome do } \\ & \text { piriforme }\end{array}$

Hongwen S, 2003 Clinical Observation on J Tradit Chin Med.

Acupuncture Treatment of Piriformis Syndrome.

Rocha TBX, Análise Comparativa Rev Bras Cien e Mov

Vilela Junior GB, Eletromiográfica do

Martins GC, Reto Femoral em

Isometria na Posição 


\begin{tabular}{|c|c|c|}
\hline $\begin{array}{l}\text { Manzatto L, } \\
\text { Grande AJ, } 2012\end{array}$ & 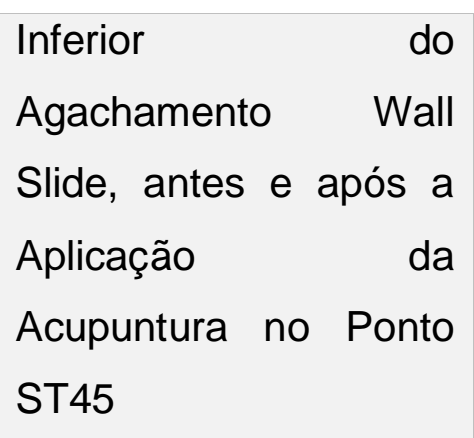 & \\
\hline $\begin{array}{l}\text { Pinheiro RG, } \\
\text { Mejia DPM, } 2012\end{array}$ & $\begin{array}{l}\text { Efeito da Acupuntura na } \\
\text { Melhora do Paciente } \\
\text { com Quadro Álgico de } \\
\text { Lesão de Menisco } \\
\text { Medial. }\end{array}$ & Monografia \\
\hline $\begin{array}{l}\text { Nunes EA, Mejia } \\
\text { DPM, } 2012\end{array}$ & $\begin{array}{lr}\text { Tratamento } & \text { de } \\
\text { Acupuntura } & \text { para } \\
\text { Combater Dores nos } \\
\text { Ombros. }\end{array}$ & Monografia \\
\hline Tolentino F, 2016 & $\begin{array}{l}\text { Efeito de um } \\
\text { Tratamento com } \\
\text { Auriculoterapia na Dor, } \\
\text { Funcionalidade e } \\
\text { Mobilidade de Adultos } \\
\text { com Dor Lombar } \\
\text { Crônica. }\end{array}$ & Dissertação \\
\hline
\end{tabular}

\section{L'ACUPUNCTURE DANS LA PRÉVENTION DES BLESSURES}

La prévention des blessures dans le sport est une grande bataille à gagner par les professionnels qui travaillent avec les athlètes. Dans le football, les blessures musculaires correspondent à $20-40 \%$ de toutes les blessures sportives, principalement $80-90 \%$ dans les membres inférieurs. ${ }^{15}$

Les blessures musculaires est le grand méchant de tous les athlètes, de divers sports, et peut les laisser loin de leurs activités pendant une longue période. II est donc 
intéressant de présenter une proposition d'intervention prophylactique, apportant l'idée d'une technique pour prévenir ou réduire les risques de ce type de blessure chez les athlètes. Dans ce cas, l'acupuncture s'avère être une technique bénéfique.

Dans son étude, Dias Junior 16, a été assisté par 54 athlètes masculins, appartenant à une équipe de football professionnel. Ils ont été répartis en 6 groupes : groupe 1 acupuncture pour rééquilibrer le système, trouvé par l'évaluation ; groupe 2 - qui a reçu un protocole avec des points spécifiques : pancréas à la rate 3 , pancréas à la rate 6 (figure 1A) et estomac 36 (figure 1B); groupe 3 - estomac 36 (figure 1B); groupe 4 pancréas à la rate 6 (figure 1A); groupe 5 - acupuncture de l'oreille; groupe de contrôle - n'a reçu aucune intervention. Après huit séances, une par semaine, les résultats ont montré : les groupes 1 et 2 ne présentaient aucun type de blessure; groupe $4:$ deux lésions; groupes 3 et $5: 1$ lésion présentée; contrôle : a présenté 6 cas de problèmes de muscle. 
Figure 1 A: pancréas de rate 3 - du côté médial du pied, posttero-inférieur de l'articulation métatarsienne-phaline, dans la ligne de la jonction de la peau foncée et claire; pancréas rate 6: 3 tsun au-dessus du maleolus médial, sur le bord postérieur du tibia; B: estomac 36 - 3 tsun sous la rotule entre le muscle antérieur du tibia et le long muscle extenseur des doigts; estomac 37 - 3 tsun en dessous de Zusanli (E36), sur le côté antérieur latéral du muscle tibia; estomac 38 - 8 tsun sous le genou, 2 tsun au-dessous du Point Shangjuxu (E37), sur le côté du muscle antérieur du tibia; estomac 41 - au point médian dorsal de la cheville au-dessus du ligament croisé, entre les tendons du long muscle extenseur hallux et le long extenseur des doigts.

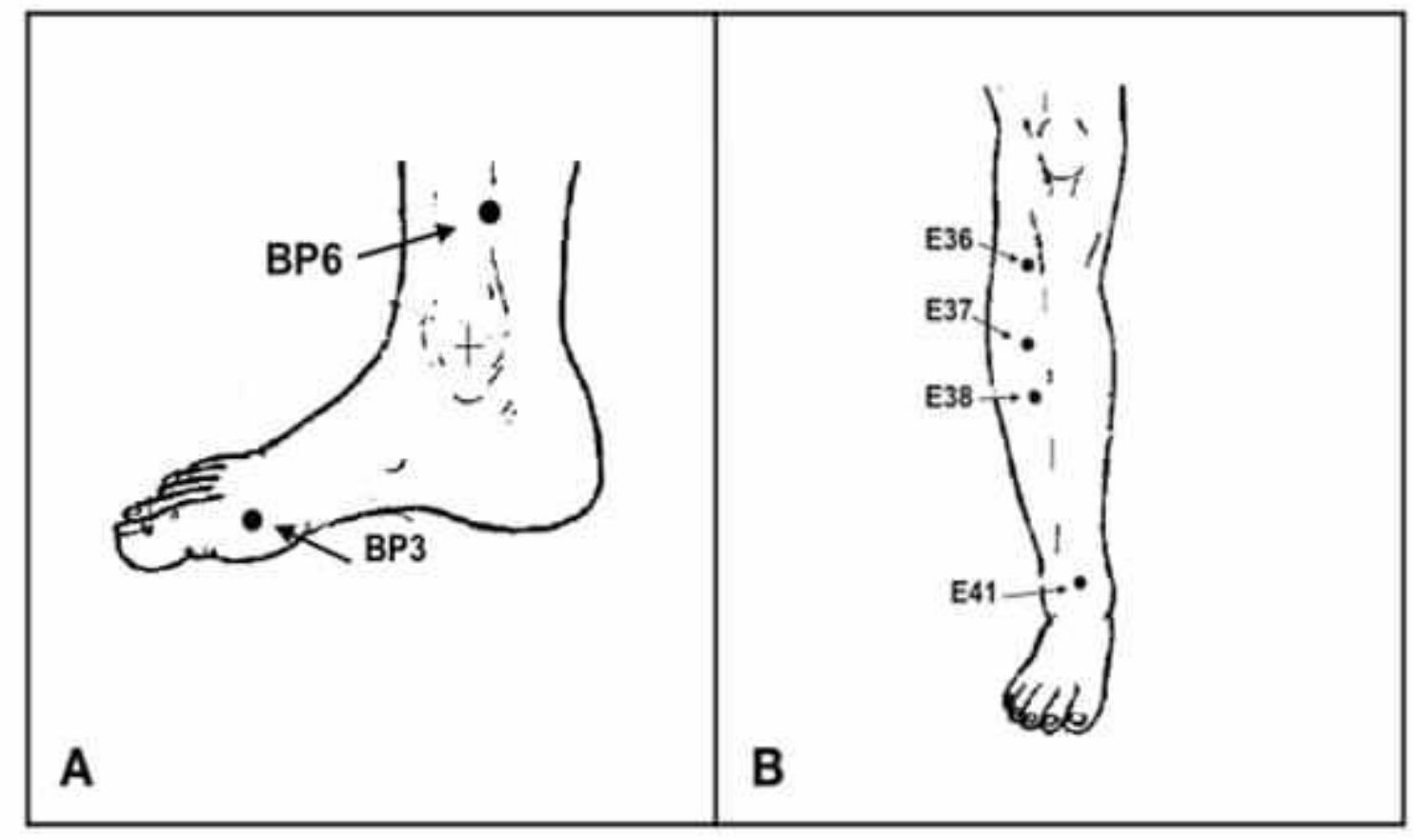

Source: Wen ${ }^{17}$

La rate du pancréas a pour fonction d'extraire l'énergie de la nourriture, de distribuer au corps et de contrôler le sang dans les vaisseaux sanguins. Le Qi de présentation fort sera transporté à tous les muscles dans le corps, spécifiquement aux membres, mais si le Qi est dans l'insuffisance, l'énergie ne sera pas transmise au tissu de muscle et l'athlète présentera la fatigue et les muscles affaiblis. ${ }^{18}$

Selon Ross ${ }^{19}$, les points de rate pancréas 3 et 6 sont associés à l'estomac 36 par tonifier le sang Qi. L'élément terre est responsable de l'équilibre énergétique et de la 
disponibilité du Qi et du sang comme sources d'énergie pour l'activité mentale, émotionnelle et physique. LE MTC, rapporte qu'en tonitant cet élément, il maintient les muscles nourris par Qi et Xue (sang dans le MTC), visant à améliorer les performances athlétiques. Cela maintient l'obligation de tonifier l'organe qui produit Xue (Liver) et maintient le liquide énergétique (pancréas et rein de la rate), puisque la fonctionnalité du sang est de nourrir le corps, en plus de compléter l'action nutritive de Qi.

L'entraînement musculaire, la relaxation et la force contractile sont liés à la nutrition du foie xue. Si la nutrition est déficiente, les crampes et la paresthésie des membres causeront des blessures. D'autre part, la rate surveille la xue à l'intérieur des vaisseaux et élimine le qi de la transmission des aliments au corps: Fort Qi, va transmettre une bonne énergie aux muscles; un Qi affaibli se traduira par un muscle fatigué et faible. 19,20

Sur la base de ces preuves, cette technique est pratiquée dans les sports présentant des résultats rapides, améliorant la qualité de la force musculaire, les conditions cardiorespiratoires, la flexibilité et le bien-être mental et physique des sportifs. ${ }^{20,21,22}$ Yang et 22 employés, ont réalisé une augmentation significative de la force musculaire, fournissant l'amélioration de la vitesse des athlètes. À son tour, Luna et ses collaborateur ${ }^{5} 24$, a déclaré que la stimulation des points d'acupuncture améliore les performances sportives, la plasticité musculaire prévenir les blessures.

Une étude menée au Japon met en évidence l'effet de l'acupuncture sur le bien-être physique des joueuses de soccer pendant la compétition. Ils ont été divisés en deux groupes, où l'un a été soumis au traitement et l'autre contrôle. Les niveaux de cortisol (évaluation du système endocrinien) et l'évaluation du bien-être physique ont été évalués à l'aide du questionnaire POMS (évaluation de l'état physique et mental) : une amélioration du système immunitaire a été observée ( sécrétion sigune diminuée), amélioration du stress mental (diminution du cortisol) et amélioration du bien-être physique, de la flexibilité et de la tension musculaire. ${ }^{25}$

Cependant Ehrlich et 25 employés, a réalisé une amélioration de la performance athlétique, l'augmentation du niveau de bien-être physique, l'amélioration de la 
réponse compétitive, pendant l'entraînement et les jeux, aider à la récupération de la capacité musculaire.

D'autres lésions qui peuvent être évitées avec l'acupuncture sont des entorses de cheville, qui affectent les ligaments, déclenchant beaucoup de douleurs et la limitation de la fonction commune. France ${ }^{26}$, a utilisé les points ashi (points douloureux à la palpation), associés à la vessie 60 (figure $2 \mathrm{~A}$ ) pour éliminer la douleur; la vésicule biliaire 39 qui renforce les os, les tendons et combat l'algie dans les extrémités; et la vésicule biliaire 40 stimule $\mathrm{Qi}$ et xue; l'estomac 41 (figure 2B) qui traite les changements latéraux de la cheville et de l'estomac 36 (figure 1B) éliminant l'obstruction et stimulant la circulation du Qi et de la xue locale.

Figure $2 \mathrm{~A}$ : vessie 60 - entre le tendon d'Achille et le bord du soleolus latéral de la cheville, au niveau du point le plus élevé du maleolus; B: vésicule biliaire 39 - 3 tsun au-dessus du point le plus élevé du maleolus externe, dans la dépression entre le péroné et les tendons fibulaires longs et courts et la vésicule biliaire 40 - sur le côté antéro-inférieur du maleolus externe, dans la dépression latérale du tendon du long muscle numérique d'extenseur ; estomac 41 - au point médian dorsal de la cheville audessus du ligament croisé, entre les tendons du long muscle extenseur halux et le long extenseur des doigts.

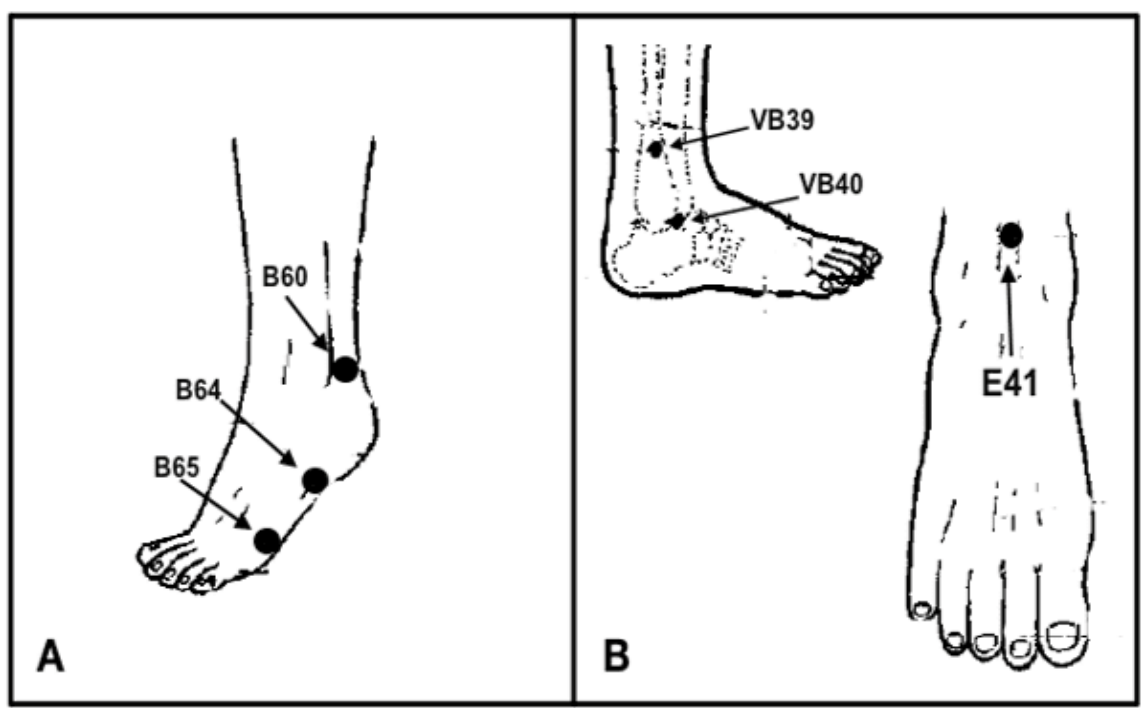

Source: Wen ${ }^{17}$ 
Certains athlètes peuvent être surpris par une entorse, qui est définie comme une blessure au tendon cervical qui provoque des douleurs musculaires et la raideur. II existe un protocole pour remédier à ce problème et le renvoyer à ses fonctions. Wadsworth ${ }^{27}$, utilisé en plus des points ashi qui stimulent la circulation de Qi et xue, la vésicule biliaire 39 (figure $2 \mathrm{~B}$ ), la vésicule biliaire 20 actes dans la circulation de Qi et xue tendons, détendre et adoucir la douleur; gouverneur ousse 14 expulser le facteur pathogène et réglementer le méridien Qi; vessie 10; intestin grêle 14; la vésicule biliaire 21 (figure 3A) stimule la circulation du Qi et du xue. Si le bloc articulaire limite la flexion et l'extension, utilisez le point de vessie 60 (figure 2A). Les points d'intestin grêle 3, poumon 7 favorisent la circulation du Qi et du xue du cou, s'il a la limitation de rotation, emploient le point 7 d'intestin grêle qui draine le méridien de Taiyang de la main (figure 3B). 
Figure $3 \mathrm{~A}$ : vésicule biliaire 20 - sous le bord occipital dans la dépression entre les muscles trapézoïdes et sternum-clidomastoïdes, sur la marge des cheveux et de la vésicule biliaire 21 - au point équidistant entre le Dazhui (VC14) et l'acromion d'épaule, 1 tsun au-dessus du point tianilien ; vase du gouverneur 14 - au milieu entre les processus épineux; septième vertèbre cervicale et première vertèbre dorsal; vessie 10 - au niveau entre les épines de la deuxième et de la troisième vertèbre, 1,3 tsun latéral de la ligne médiane dorsale, sur le bord du muscle trapézoïde et de l'intestin grêle 14 - 3 tsun latéral de l'axe de la vertèbre; au niveau horizontal du bord inférieur du processus épineux de la première vertèbre dorsale; B: poumon 7 - sur le côté médial de l'avant-bras, 1,5 tsun au-dessus de la ligne du poignet entre les tendons du long muscle adducteur du pouce et le long muscle extenseur carporadial; intestin grêle 3 côté ulnaire de la main, derrière l'articulation métacarpienne-phalangienne du cinquième doigt, entre les peaux légères et foncées; Petit intestin 7 - 5 tsun au-dessus du poignet, sur le côté ulnaire du muscle extenseur carpal-ulnaire.

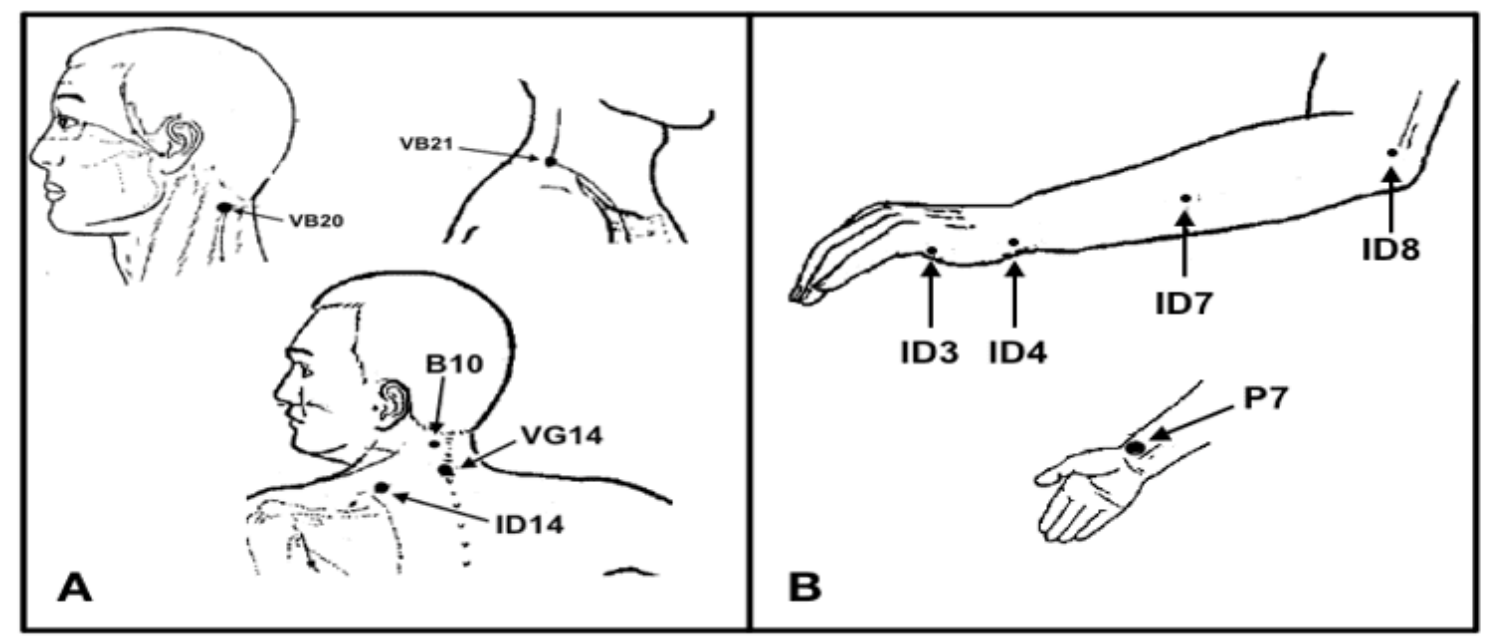

Source: Wen ${ }^{17}$

Surcharger l'articulation du coude, affecte, en particulier les joueurs de tennis, et peut conduire à un blocus de Qi et xue, déclenchant une inflammation chronique appelée épicondilite. Les points Ashi sont utilisés dans la région pour promouvoir la stimulation Qi. 28 
Pour le traitement des lésions du poignet, telles que: tendinite ou ténosynovite du pouce, extenseurs et fléchisseurs des doigts, France 26, a utilisé le point ashi associé au gros intestin 5 pour disperser la chaleur du méridien; l'intestin grêle 5 éliminant la chaleur et déverrouillant le poignet (figure 4A); Triple chauffe-eau 4 qui, en plus d'éliminer la chaleur cesse l'inflammation et le gros intestin 11 (figure 4B) déverrouillage qi et xue calmer la douleur.

Figure $4 \mathrm{~A}$ : intestin grêle 5 - du côté ulnaire du poignet, dans la dépression entre le pisiforme et le processus ulnaire styloid; Gros intestin 5 - sur le côté arrière-radial du poignet, un peu distal de l'os radio, où il ya une dépression entre les tendons du muscle extenseur court et long du pouce, lors de l'étirement et l'ouverture du pouce. B: triple chauffe-eau 4 - du côté dorsal du poignet, dans la dépression au milieu du pli dorsal du poignet, entre les tendons des muscles extenseurs numériques communs et extenseur numérique du cinquième doigt;gros intestin 11 - sur le côté radial du coude, dans le muscle brachioradial; lors de la flexion du coude, dans la dépression radiale à la fin de la ligne cubitale.

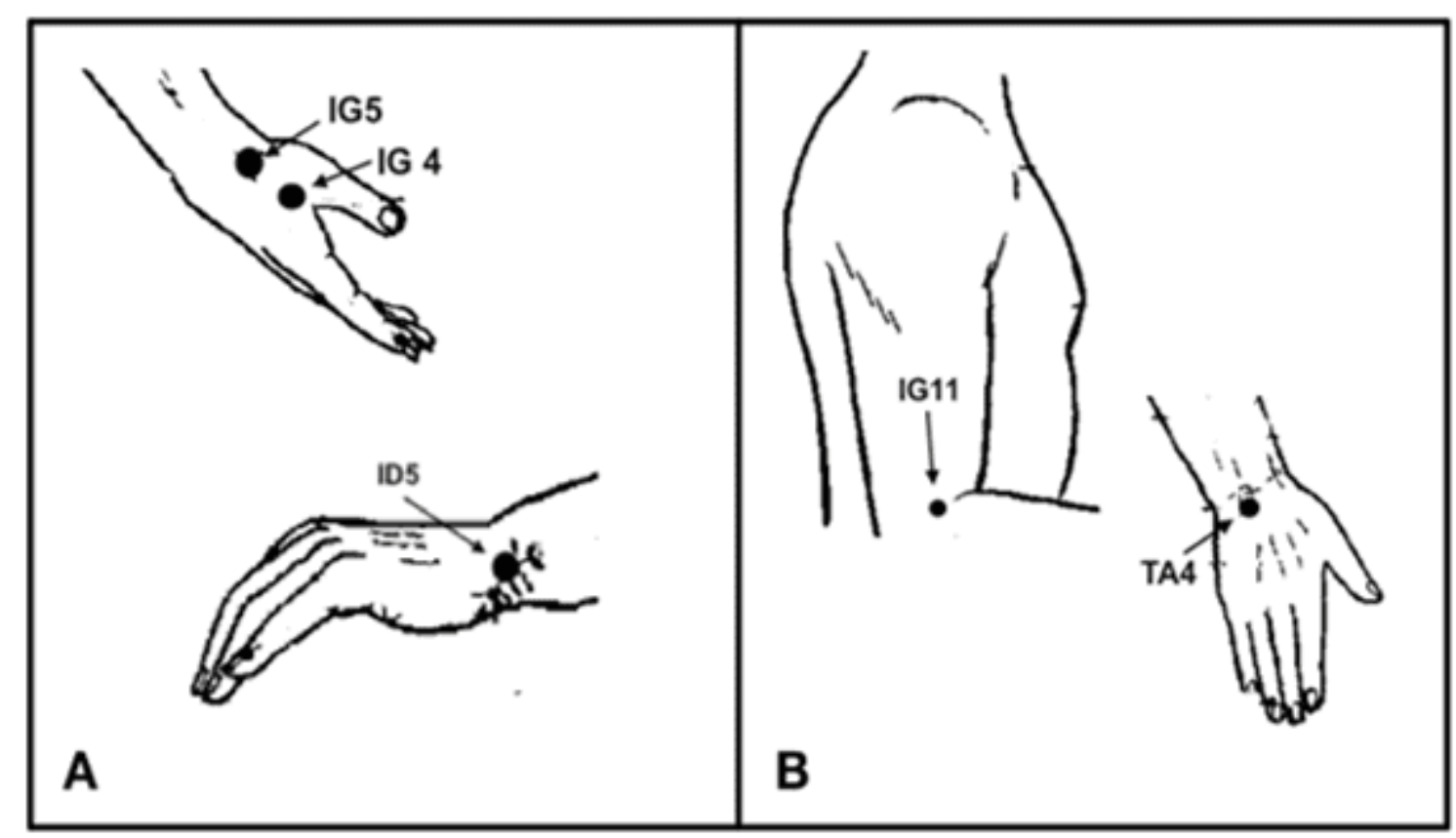

Source: Wen ${ }^{17}$ 
Pour les lombalgies, Barela 17, a associé de ${ }^{\mathrm{s}}$ points ashi à la vessie 23 et à la vessie 25 pour stimuler le Qi et le Xue locaux, la vessie 40 (figure 5) qui élimine la douleur et la chaleur.

Figure 5 : vessie 23 - 1,5 tsun, latérale du bord inférieur du processus spinal de la vertèbre (L2); vessie 25 - 1,5 tsun, latérale du bord inférieur du processus spinal de la vertèbre (L4); vessie 40 - 3 tsun, latérale de l'axe (Du-Mai), au niveau de bord inférieur dans la deuxième colonne vertébrale de la vertèbre (T2).

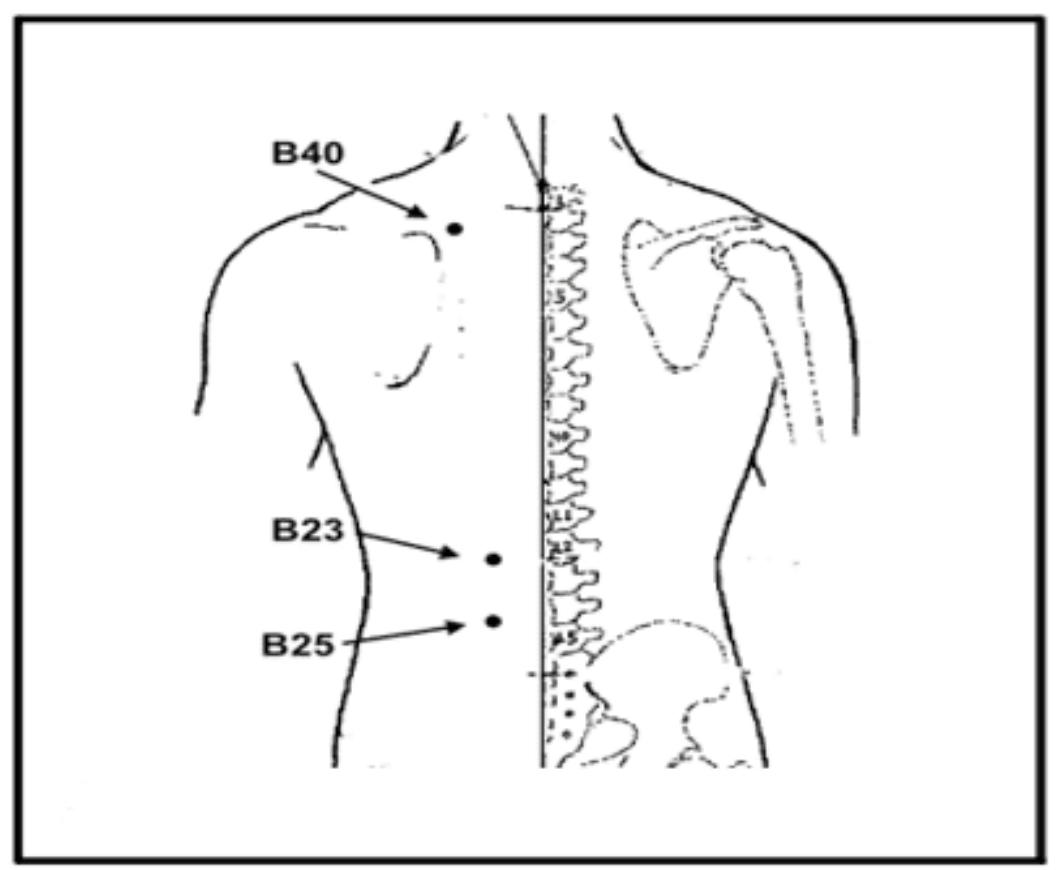

Source: Wen ${ }^{17}$

Maintenant, une autre blessure qui inquiète les athlètes de football, sont les ligaments, les ménisques et les tendons du genou. Ceci sont traités avec des points d'ashi, associés à la vésicule biliaire 33 (figure 6) qui détend des tendons et traite la douleur ; la vessie 40 (figure 5) élimine les douleurs articulaires, l'œdème et la chaleur, stimulant Qi et xue; l'estomac 36 (figure 1B) régulant le flux de Qi et de xue du méridien éliminant l'obstruction. ${ }^{29}$ 
Figure 6: vésicule biliaire 33 - au bord latéral du genou, 3 tsun au-dessus du point yanglingquan (VB34), dans la dépression supérieure de l'épithène latéral du fémur.

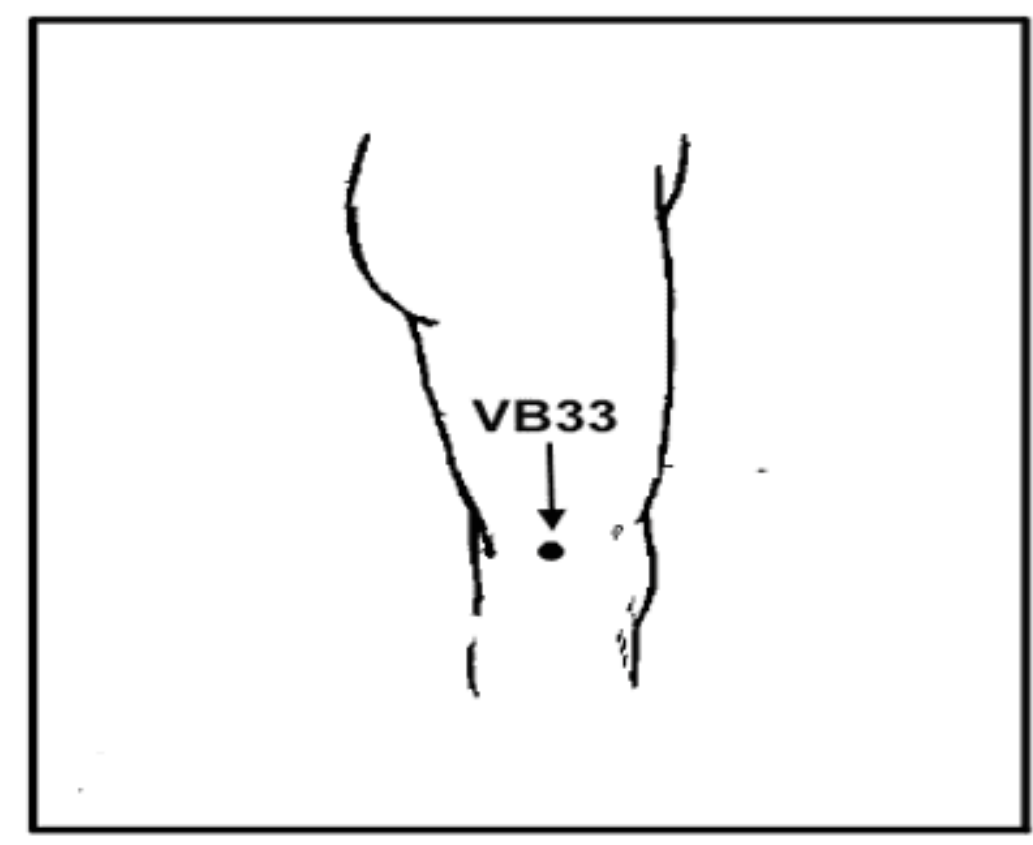

Source: Wen ${ }^{17}$

Gemeo ${ }^{30}$, a déclaré que l'acupuncture peut favoriser une amélioration de la capacité physique des athlètes, prévenir et traiter les blessures qui peuvent survenir avant, pendant ou après les compétitions sportives, en plus d'aider et d'influencer des facteurs émotionnels tels que l'anxiété, l'irritabilité, l'insomnie et la dépression, qui peuvent directement entraver la capacité athlétique de ces personnes. Il a développé un protocole spécifique en utilisant des points d'acupuncture maître, en sélectionnant en fonction de la modalité (graphique 1):

Tableau 2 : Gemeo ${ }^{30}$

\section{Maître de l'énergie vase gouverneur 19 (figure 7) morale}




\begin{tabular}{|c|c|}
\hline $\begin{array}{l}\text { Maître de l'énergie } \\
\text { générale }\end{array}$ & $\begin{array}{l}\text { influence l'énergie générale, agissant sur l'énergie } \\
\text { ancestrale et postnatale - vase du gouverneur 4, 6, 10, } \\
13 \text { (figure 7) }\end{array}$ \\
\hline $\begin{array}{l}\text { Maîtres des membres } \\
\text { supérieurs }\end{array}$ & $\begin{array}{l}\text { agit sur les mouvements de l'avant-bras, les poignets, } \\
\text { les mains et les épaules : triple chauffe-eau } 15 \text { (figure } 7 \text { ) }\end{array}$ \\
\hline des poumo & inter \\
\hline ère & $\begin{array}{l}\text { diaphragme, la respiration et nn. phrénique : vessie17 } \\
\text { (figure } 7 \text { ) }\end{array}$ \\
\hline le ventre & ille abdominale \\
\hline ins & $\begin{array}{l}\text { actes de taille lombaire : vessie } 47 \text { et vessie } 52 \text { (figure } \\
\text { 8) }\end{array}$ \\
\hline Maître de hanche & rs et articulation \\
\hline sse & $\begin{array}{l}\text { actes dans la musculature des cuisses : vésicule biliaire } \\
30 \text { (figure 8) }\end{array}$ \\
\hline $\begin{array}{l}\text { Maître de membre } \\
\text { inférieur }\end{array}$ & $\begin{array}{l}\text { influence l'action des pieds et des muscles en général : } \\
\text { vessie } 58 \text { (figure } 8 \text { ) et estomac } 36 \text { (figure 1B) }\end{array}$ \\
\hline $\begin{array}{l}\text { Points pour améliorer } \\
\text { l'équilibre et la réflexion }\end{array}$ & $\begin{array}{l}\text { vaso conception } 4 \text {, vaso conception } 6 \text { (figure 9), gros } \\
\text { intestin } 11 \text { (figure } 4 \mathrm{~B} \text { ), vessie } 46 \text { (figure } 8 \text { ), vaisseau- } \\
\text { gouverneur } 4 \text { (figure } 7 \text { ). }\end{array}$ \\
\hline
\end{tabular}

Figure 7 : vase du gouverneur 4 - dans la ligne centrale de la colonne vertébrale; dans l'espace entre les épines des deuxième et troisième vertèbres lombaires; vase gouverneur 6 - dans la ligne centrale de la colonne; entre le onzième et le xiie processus épineux des vertèbres dorsales; vase gouverneur 10 - dans la ligne centrale de la colonne; entre le sixième et le septième processus épineux des vertèbres dorsales; vase du gouverneur 13 - dans la ligne centrale de la colonne; entre le premier et le deuxième processus épineux des vertèbres dorsales; vase du gouverneur 19 1,5 tsun derrière Baihui (VG 20); estomac 27 - 2 tsun sous le nombril et 2 tsun à côté de la ligne médiane sur le bord latéral du muscle rectus-abdominal; vessie 17 - 1,5 tsun de l'axe, au niveau inférieur du bord du processus spinal de la vertèbre (T7); poumon 1 - sur le côté antérolatéral de la poitrine, en dessous du point Yunmen (P2) (pit entre la clavicule et l'épaule), dans l'espace entre la première et la deuxième côte, 
6 tsun sur le côté de la ligne médiane du corps; triple chauffage 15 - dans la fosse suprascapulaire, entre Quyuan (ID13) et Jianjing (VB21).

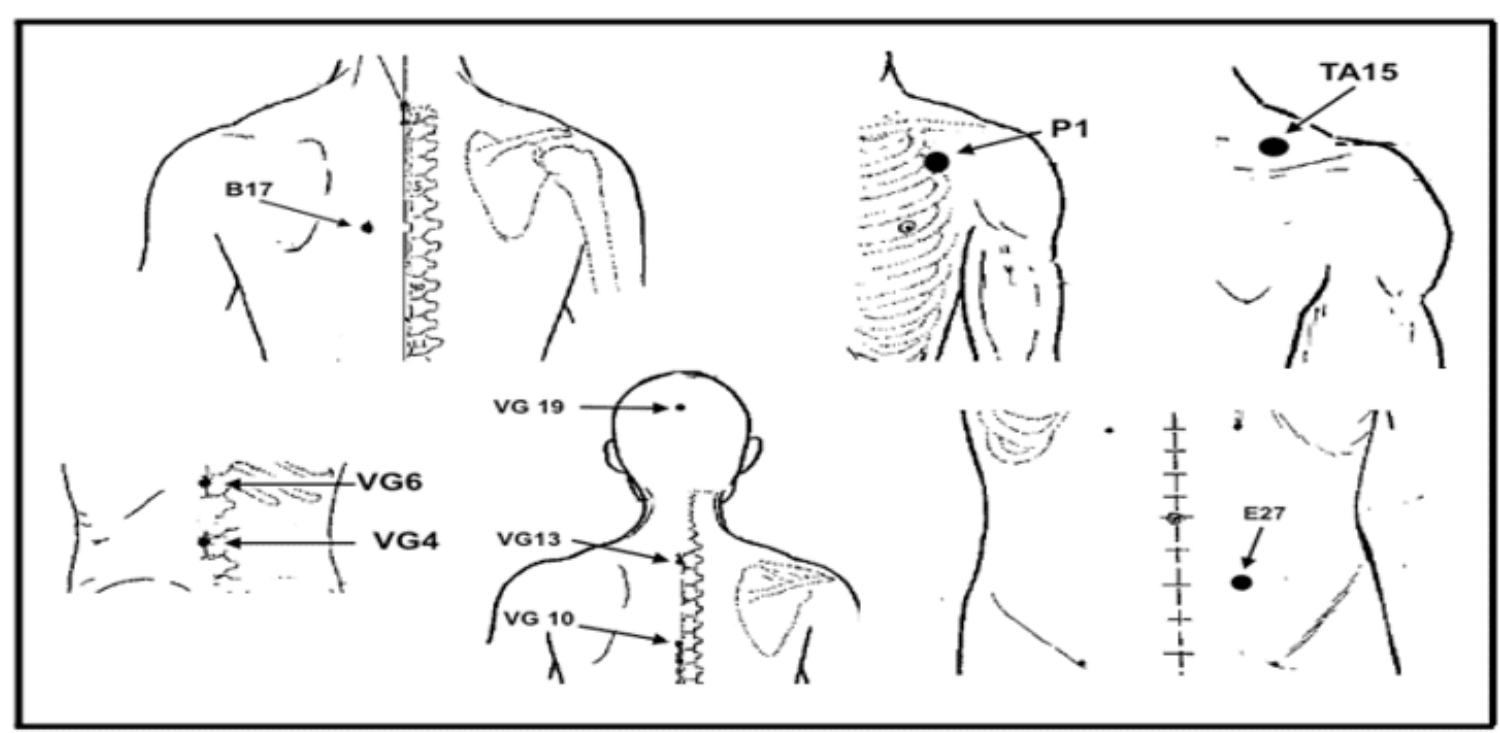

Source: Wen 17 
Figure 8 : vessie 46 - 3 tsun, latérale de l'axe, au niveau inférieur de la colonne vertébrale de la vertèbre (T9); vessie 47 - 3 tsun, latérale de l'axe, au niveau inférieur de la colonne vertébrale des vertèbres (T10); vessie 52 - 3 tsun, latéral de l'axe, au niveau de la deuxième colonne vertébrale du sacrum, au bord latéral de l'articulation iliossacral; vessie 58 - I tsun sous le côté du point chengshan (B57); 7 tsun au-dessus du talon, sur le côté latéral du tendon musculaire gastrocnemius; vésicule biliaire 30 dans la fesse, dans la ligne entre le hiatus sacré et l'enavant du plus grand trochanter; un tiers de la distance latérale au bord inférieur du muscle piriforme; point de hanche supplémentaire - au milieu de la ligne allant de l'extrémité supérieure de la rainure interglutée à la partie de la crête extérieure de l'ischio.

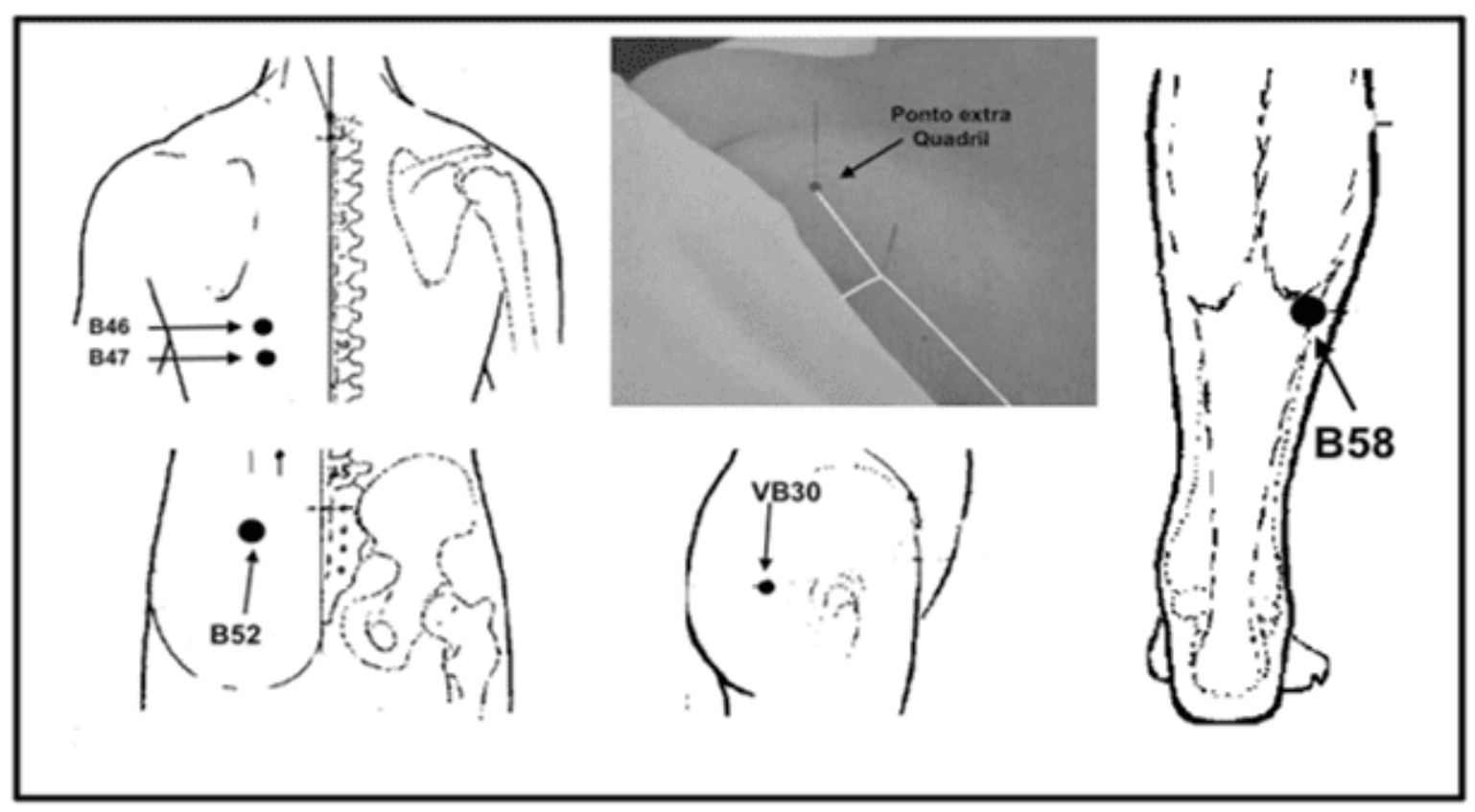

Source: Wen ${ }^{17}$; Rosseto ${ }^{31}$ 
Figure 9 : conception de vase 4 - 3 tsun sous le nombril, dans la ligne centrale de l'abdomen ; conception de vase 6 - 1.5 tsun sous le nombril, dans la ligne centrale de l'abdomen.

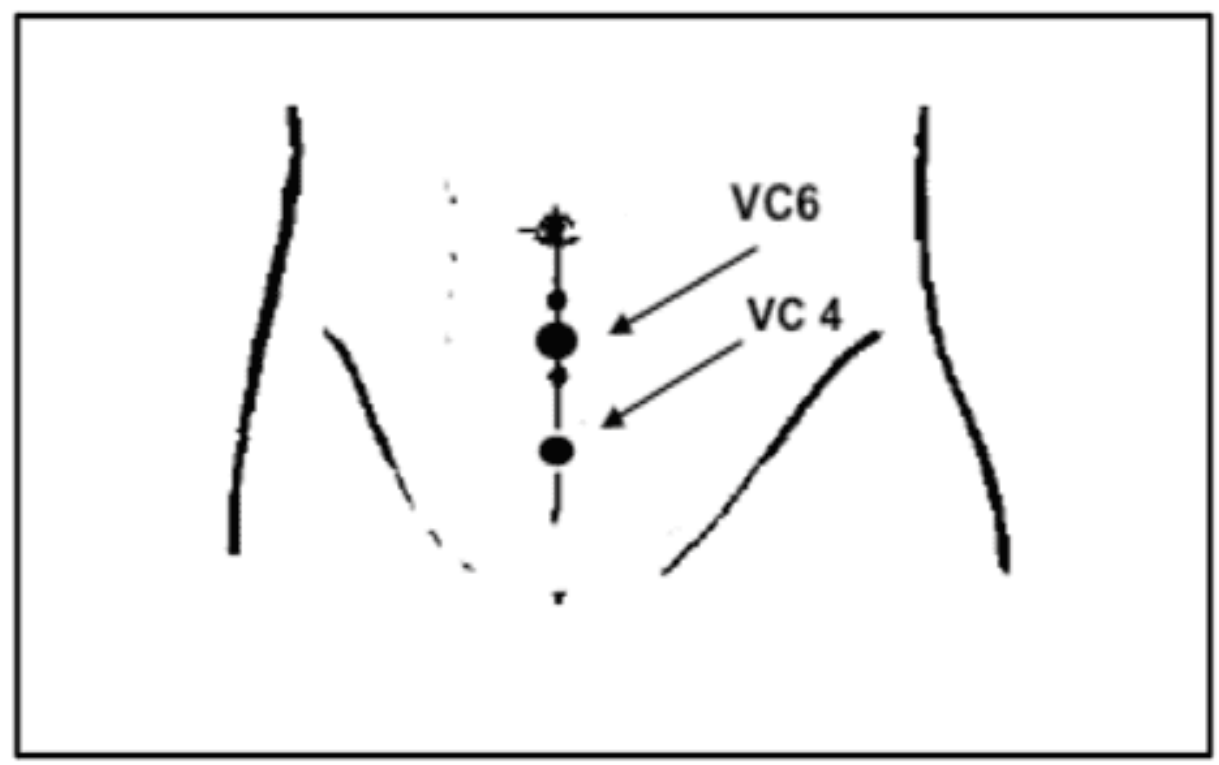

Source: Wen ${ }^{17}$

\section{L'ACUPUNCTURE DANS L'AMÉLIORATION DE LA PERFORMANCE ATHLÉTIQUE}

Lorsqu'il s'agit de l'acupuncture dans la prévention des blessures chez les athlètes, nous ne devons pas oublier que la réalisation de cet objectif préventif, par conséquent, nous améliorer la qualité et l'intégrité physique et peut intervenir dans l'amélioration des performances.

Dans les pays asiatiques, l'utilisation de l'acupuncture développe un bien-être physique pour les athlètes, régulé par le système immunitaire nerveux, endocrinien et, par conséquent, une amélioration de la condition physique dans plusieurs modalités. 32, 31, 34 En outre, il ya des points appliqués avant, pendant et après l'entraînement et les jeux, qui aident à l'entretien des muscles, tels que la réduction de l'acide lactique, la réduction de la douleur, la fatigue, l'accélération de la récupération et l'amélioration des performances. ${ }^{35}$ 
L'acupuncture stimule la libération de substances biochimiques qui soulage la fatigue musculaire des exercices intenses, en plus de promouvoir une activité antioxydante accrue, diminuant les taux de stress oxydatif. Au cours d'une étude, les athlètes ont reçu un traitement par électroacupuncture pendant 30 minutes, sur une période de 30 jours et le résultat a été l'augmentation significative de la dismutase de superoxyde (SOD) et de la diminution du malondial sanguin (MDA). La dismutase de superoxyde est une enzyme avec des effets antioxydants qui protège des cellules contre des substances toxiques en exerçant la réponse anti-inflammatoire puissante dans le corps. Le MDA est lié aux maladies inflammatoires et dégénératives, permettant aux chercheurs de conclure que l'électroacupuncture diminue les taux de MDA et augmente soD, contribuant au soulagement de la fatigue et l'amélioration de la performance physique. ${ }^{36}$

Bien qu'il s'agit d'une technique millénaire, son utilisation améliore la performance physique et mentale de l'athlète est récente. Luna, a publié une recherche démontrant que l'acupuncture a statistiquement amélioré la force maximale et la puissance des athlètes sprinter (100, 200 et 400 mètres peu profonds sans barrières) et depuis lors la recherche à cet égard a été approfondie. ${ }^{37}$

Dans une recherche, $S^{\text {antos } 20}$ a utilisé des athlètes masculins Handbol entre 15 et 17 ans, où il a effectué deux coups de 100 mètres dans chaque athlète (pré acupuncture). Après 45 minutes d'application du protocole choisi pour le traitement, il a réévalué de la même manière. Les points choisis étaient les suivants : vessie 58 (figure 8) maître des jambes et du pied; l'estomac 36 (figure 1B) tonifie la musculature striée et est utilisé pour le traitement du potentiat; le point principal supplémentaire des hanches (figure 8) tonifie les fessiers et augmente la flexibilité de l'articulation coxofemoral; vésicule biliaire 30 (figure 8 ) maître des cuisses et favorise la flexibilité pour le mouvement des membres inférieurs; le maître des poumons du poumon 1 (figure 7) favorise un travail respiratoire efficace; vessie 17 (figure 7) maître du dos, diaphragm shu dorsal et point d'influence de Xue qui régule le rythme respiratoire; triple plus chaud 15 (figure 7) maître des bras qui commande le groupe musculaire articulaire 
des épaules, de la nuque, de la colonne cervicale, des six premières vertèbres thoraciques, des bras, des avant-bras, des poignets et de la main.

Les résultats ont permis d'identifier une amélioration significative, avec une diminution du temps dans la deuxième batterie des essais de 100 mètres. L'amélioration moyenne dans les temps était de $5,72 \%$ et l'athlète avec la meilleure performance atteint atteint atteint un pourcentage de 10,84\%.

Cette moyenne de 5,72\% atteint dans l'amélioration de la performance semble faible, mais si on le compare aux résultats des compétitions importantes, nous pouvons voir la validité de la pratique: aux Jeux panaméricains de Rio de Janeiro 2007, dans la finale du 100 mètres peu profond mâle à différence entre la 1ère place et la 8e place était de 0,23 seconde, ce qui représente un temps de 2,26\% plus élevé, c'est-à-dire que si cet athlète réalisait une amélioration de $5,72 \%$ de sa performance, il obtiendrait probablement d'autres résultats.

Il ya peu d'attention scientifique, lorsque nous traitons, des effets d'acupuncture sur les améliorations de la réponse physiologique induite par l'exercice et peu dirigé vers les athlètes de haute performance. ${ }^{24,38}$ Mais Rossetto ${ }^{31}$ présente un protocole pour améliorer la performance athlétique dans le football et le rugby en utilisant les points suivants: vessie 52 et vessie 58 (figure 8); vessie 17 et poumon 1 (figure 7 ).

Le point de la vessie 58 , tonifie la musculature strié de tout le corps, l'articulation des genoux, des chevilles et des pieds. C'est un point important pour éviter la fatigue, surtout si elle est utilisée avec l'estomac 36. La vessie 17 tonifie les muscles dorsaux, les ligaments articulaires et augmente même la flexibilité des articulations vertébrales elles-mêmes, favorisant une action réglementaire sur le diaphragme, le rythme respiratoire et les nerfs phréniques. La vessie 52 tonifie toute la région des muscles lombaires et favorise la volonté. Le poumon 1 est utilisé pour rendre le travail respiratoire efficace et est lié aux nerfs supraclavaires et intercostal (avec une insertion plus profonde) aidant au plexus brachial. L'utilisation avec le point de vessie 17 régularise le rythme respiratoire. 
Comme Bopp Limoge 39, a appliqué un protocole fixe en trois points: foie 5 (figure 10), triple chauffe-eau 15 (figure 7) et vessie 58 (figure 8), ajouté à deux autres points aléatoires, placebo, chez 35 athlètes, la réalisation d'une amélioration significative de la performance athlétique des personnes testées.

Figure 10 : foie 5 - 5 tsun au-dessus du maleolus médial ; au bord postérieur du tibia.

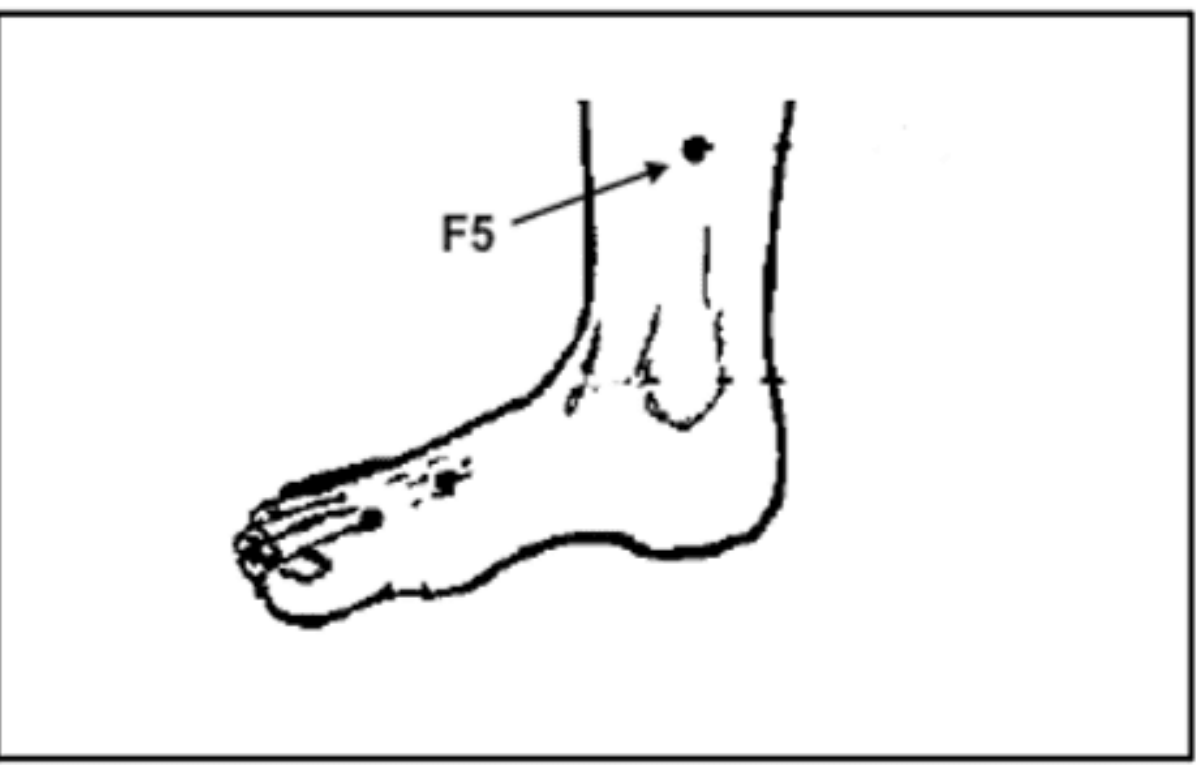

Source: Wen ${ }^{17}$

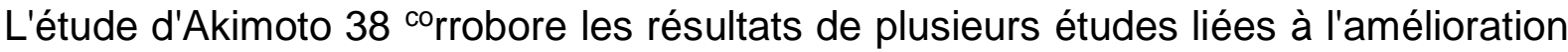
de la capacité physique et mentale, où il a testé vingt et un athlètes de football pendant la phase de compétition, évaluant biochimiquement l'IgA salivaire et le cortisol, par taux tension et fatigue de muscle, ayant pour résultat le cortisol accru et l'IgA salivaire inférieur, confirmant l'amélioration efficace de la performance athlétique et mentale.

Dans une autre recherche menée dans le traitement des blessures sportives avec l'acupuncture cinétique (association de l'acupuncture avec kinésiothérapie), chez les athlètes de tennis, soccer, volley-ball, gymnastique olympique, capoeira, balle et jiujitsu; Patients présentant des désordres locomoteurs tels que : tendinite d'Achille, douleur dorsale, douleur dorsale, prarcicolo, tenosynovitis, syndrome de tunnel de carpal, syndrome de pronador, contusion de muscle, coude de tennis et gonalgia dû 
aux dommages de ménisque. Les 31 athlètes testés ont repris l'entraînement, tout au plus, jusqu'à la troisième séance : $53 \%$ sont revenus après la première séance; $37 \%$ après la seconde; $11 \%$ après le troisième, concluant que cette technique a agi efficacement en accélérant le temps de récupération des athlètes. L'explication de ceci est que l'acupuncture agit dans l'inhibition du cycle de spasme - douleur, menant à un bloc afferent segmentaire, bloc descendant supraspinal, par des voies pyramidales et l'activation des analgésiques endogènes de processus. ${ }^{26}$

Dans une autre étude, les variables de la force maximale, de la force explosive, de la résistance anaérobie et de la vitesse dans les couloirs de sprinter à haut rendement ont été évaluées au cours d'une période de transition. Le méridien de foie a été concentré : foie 1 ; foie 3 ; foie 8 (figure 11A), parce que les fonctions énergétiques de cet organe sont de stocker le sang, contrôler la dispersion, le drainage et de déterminer les affections tendineuses et ligamentaires, en plus de contrôler la partie émotionnelle, parce qu'un déficit énergétique peut déclencher: la dépression; irritabilité; l'insomnie; rêves troublants. Le premier acupoint perforé était la vessie 62, la vésicule biliaire 34, le rein 3 (figure 11B), suivi de la rate du pancréas 3 (figure $1 \mathrm{~A}$ ), du foie 1 , du foie 3 , du foie 8 (figure 11A), de l'estomac 36 (figure 1B) et de la fermeture avec l'intestin grêle 3 (figure 3B). Des points de foie ont été introduits dans la huitième session. Les résultats ont été positivement percutants, développant une amélioration des fonctions étudiées. ${ }^{24}$ 
Figure $11 \mathrm{~A}$ : foie 1 - 0,1 tsun au-dessus de l'angle latéral du lit d'ongle de l'orteil et du foie 3 - entre le premier et le deuxième métatarse, derrière les articulations métatarsiennes-phalangeal; foie 8 - à la fin du côté médial du pli popliteal, sur le bord antéro-médial des muscles semi-membraous et semitendineux. B: rein 3 - entre le bord postérieur du maléolus médial et le tendon d'Achille; vésicule biliaire 34 - I tsun sous le genou, dans la dépression antérieure et inférieure de la tête du péroné, dans le fascia du long muscle peroréen; bexia 62 - 0.5 tsun au-dessous du maleolus externe, dans la dépression inférieure du maleolus.

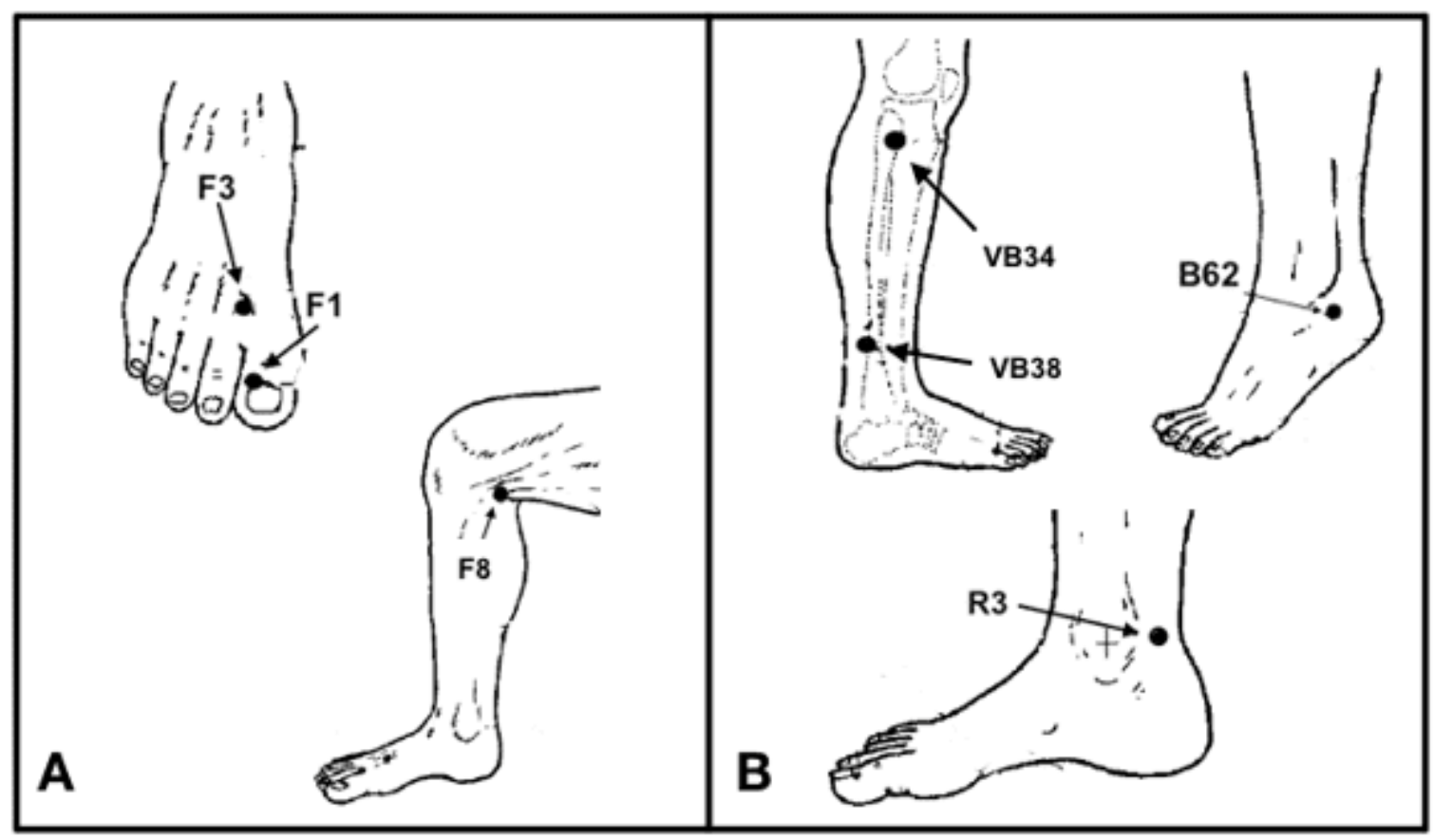

Source: Wen ${ }^{17}$

Ehrlich ${ }^{25}$, d'autre part, a divisé 36 individus masculins en trois groupes : un traité avec l'acupuncture systémique ; un autre groupe témoin; un autre avec l'acupuncture placebo. Une amélioration significative de la performance physique et des paramètres hémodynamiques a été obtenue. Une séance a eu lieu par semaine au vaso 20 du gouverneur, au vaso conception 15 et au foie 13, à la vessie 43 (figure 12), à l'estomac 36 (figure 1B) et au pancréas à la rate 6 (figure 1A) pendant 5 semaines. Ils ont analysé que le seuil anaérobie s'est amélioré de $6,62 \%$ et la performance maximale dans 
$7,15 \%$ dans le groupe qui a reçu l'acupuncture systémique, déjà dans le groupe témoin et le placebo, il n'y avait aucune altération.

Figure 12 : vase de gouverneur 20 - sur la ligne de tête centre-verticale ; 7 tsun audessus du bord postérieur des cheveux; 5 tsun derrière la marge antérieure des cheveux; vaso conception 15 - 3 tsun au-dessus de Zhongwan (RM12), dans la ligne centrale de l'abdomen et du foie 13 - au bord inférieur du point d'extrémité de la onzième côte, sur le côté de l'abdomen; vessie 43 - 3 tsun, latérale de l'axe (Du-Mai), au niveau de bord inférieur de la colonne vertébrale de la vertèbre (T5).

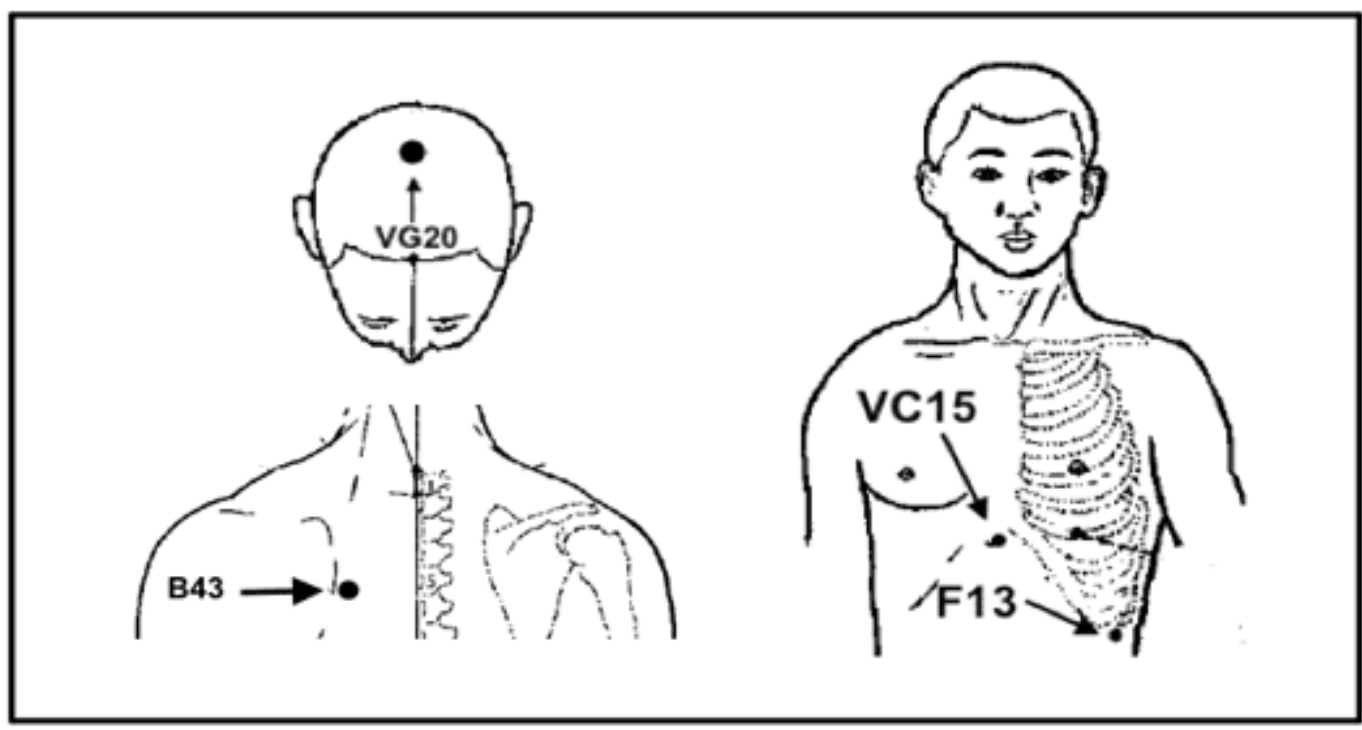

Source: Wen ${ }^{17}$

Lin ${ }^{40}$, a évalué les effets de l'acupuncture de l'oreille sur la fréquence cardiaque (Cmf), la consommation d'oxygène (VO2max) et l'acide lactique du sang chez les athlètes de basket-ball d'élite. Ils ont été divisés en 2 groupes de 12 athlètes chacun, où: Groupe 1 - acupuncture auriculaire; Groupe 2 - contrôle. Chacun des athlètes a pédalé sur un vélo d'exercice jusqu'à l'épuisement, et le Cmax, VO2max et l'acide lactique du sang ont été mesurés pendant la période de repos, après le chauffage et pendant l'exercice: après 5,30 et 60 minutes. Les résultats ont prouvé que $\mathrm{HR}$ et acide lactique de sang étaient sensiblement plus bas que dans le groupe témoin, après 30 et 60 minutes d'activité. 
Le même auteur, Lin ${ }^{41}$, dans une nouvelle recherche, a évalué les mêmes variables que FCm ' $x$, VO2max et l'acide lactique du sang dans 30 athlètes de basket-ball, divisé en 3 groupes: 1- acupuncture systémique; 2- groupe fictif; Contrôle 3. Dans le groupe 1, le point de circulation et de sexualité 6 (figure 13), l'estomac 36 (figure 1B) ont été utilisés et, au cours de l'étude, chaque athlète a effectué les tests sur un tapis roulant d'exercice pendant 15 minutes. Les variables ont été mesurées dans le repos et dans 5,30 et 60 minutes après l'exercice, obtenant une réduction significative des trois variables après 30 et 60 minutes.

Figure 13: circulation et sexualité 6 - 1 tsun en dessous du point jianshi (PC5); 2 tsun au-dessus du poignet, entre les tendons des muscles longs et le fléchisseur carpienradial

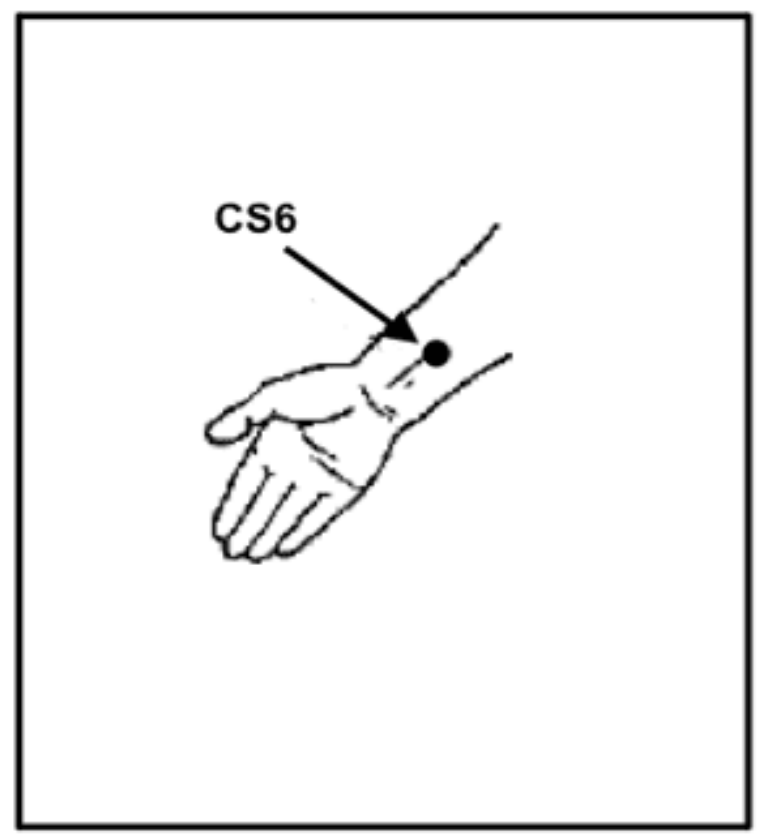

Source: Wen ${ }^{17}$

Dhillon ${ }^{42}$, aévalué la performance de 20 cyclistes masculins, subdivisés en trois groupes : traités avec l'acupuncture systémique : estomac 36 (figure 1B), vésicule biliaire 34 (figure 11B), foie 11 (figure 14), rein 3 (figure 11B), vaisseau-gouverneur 20 (figure 12 ); l'acupuncture fictive; contrôle, sans intervention. Les athlètes ont pédalé à vélo stationnaire pendant $20 \mathrm{~km}$ à leur vitesse maximale soutenue. L'échelle 
analogique de la douleur, visuelle, pour les membres inférieurs, induite par l'exercice; L'ampleur de l'effort perçu de Borg; après l'exercice des concentrations de lactate de sang. Le groupe induit par l'acupuncture a présenté un score plus élevé dans l'échelle borg, ce qui signifie que le traitement a favorisé un plus grand effort pendant l'activité et a diminué le temps de l'achèvement de l'essai.

Figure 14: foie 11 - 1 tsun en dessous de l'inginal; au bord antéro-médial du seuil du long muscle adducteur

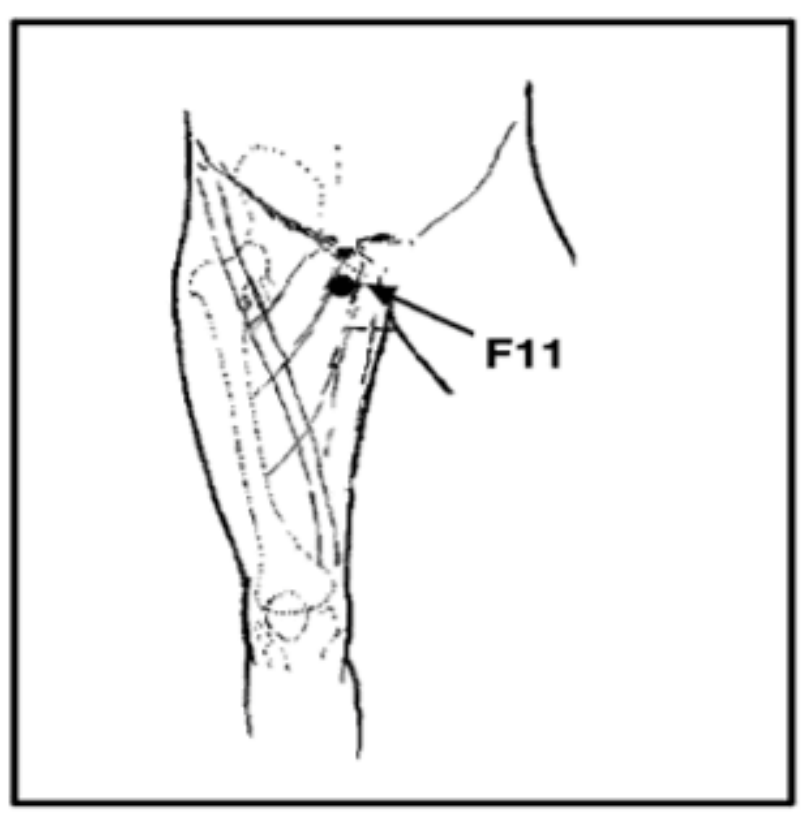

Source: Wen ${ }^{17}$

Selon Hubscher ${ }^{43}$, il a évalué le saut maximal avec l'atterrissage bipode et la force isométrique maximale volontaire du quadriceps, avec l'électromyographie de surface pour mesurer l'activité musculaire fémorale de rectus pendant 30 secondes de soutien. Trente-trois athlètes ont été divisés (13 femmes et 20 hommes) en trois groupes : acupuncture systémique à l'aide de points gastriques 36 (figure 1B), pancréas de la rate 6 (figure 1A), conception vaso 6 (figure 9); groupe d'acupuncture avec des points inexistants; Groupe de contrôle utilisé désactivé au laser. En conséquence, il s'agissait d'une augmentation non significative du saut maximal, mais d'une augmentation importante de la force isométrique des quadriceps. 
Dans une autre enquête Geng ${ }^{44}$, il a recruté 12 hommes en bonne santé avec des étudiants en éducation physique entre 19 et 25 ans, évaluant les effets de l'acupuncture de l'oreille sur les niveaux d'acide lactique du sang, induits par l'exercice sur tapis roulant ( $\mathrm{V}^{\mathrm{O}} 2 \mathrm{max}$ ). II a choisi les points : Foie, Poumon, Subcortex, Endocrine Glands et Triple Heater (figure 15). Les résultats ont été une augmentation du renforcement de la capacité d'oxygène et une diminution des niveaux d'acide lactique dans le sang après les exercices.

Figure 15 : Points d'oreille

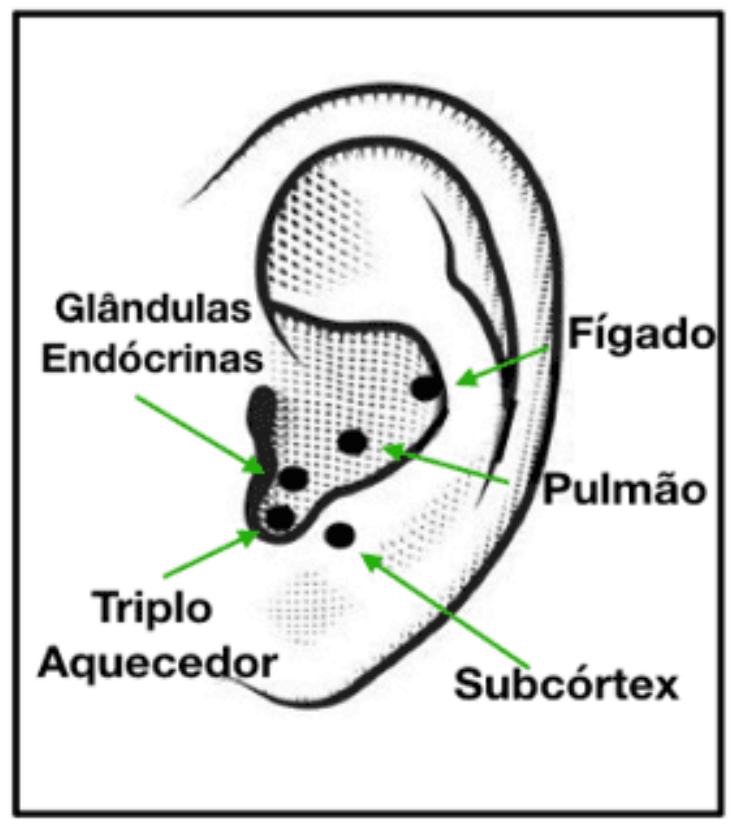

Source: Archives personnelles de l'auteur

Une autre étude de 28 individus masculins divisés en un groupe de contrôle et expérimental. Une collecte de données a été effectuée avant les séances d'acupuncture : Mesure de la fréquence cardiaque basale, à travers l'oxymètre ; mesure de la fréquence cardiaque après des tests physiques à la Harvard Bank, avec une hauteur de $50 \mathrm{~cm}$, effectuer des ascensions et des descentes pendant 5 minutes; fréquence cardiaque mesurée en 1 minute et demie du test, 2 minutes et demie et 3 minutes et demie; calcul de l'indice de condition physique (IAF); fréquence cardiaque moyenne et le nombre d'ascensions et de descentes pendant les tests de banque 
harvard. Les points ont été utilisés : poumon 10 et vaso conception 17 (figure 16), intestin grêle 3 (figure $3 \mathrm{~B}$ ), afin de tonus et conduire à la relaxation musculaire, le renforcement des tendons, la colonne lombaire et les membres inférieurs. Dans les points de vésicule biliaire 34 (figure 11B), le pancréas à la rate 4 et le rein 7 (figure 16) étaient liés à la sédation pour potentialiser le mouvement de la circulation sanguine (Xue) et de la lymphe (Jing Yie). Huit séances ont été effectuées, une par semaine et les résultats n'ont pas montré une amélioration significative par rapport à la fréquence cardiaque et la condition physique. En augmentant les performances physiques, les montées et les descentes de la Harvard Bank, les valeurs ont été significatives pour le groupe expérimental par rapport au contrôle. ${ }^{36}$

Figure 16: poumon 10 - sur le côté palmr, au-dessus de l'articulation du premier métacarpien numérique, entre les peaux sombres et légères; conception de vase 17 dans la ligne médiane du sternum au niveau du mamelon ; rein 7 - 2 tsun au-dessus de Taixi (R3); sur le bord antéro-médial du muscle de semelle; pancréas de la rate 4 sur le côté médial du pied; 1 tsun derrière l'articulation métatarsophale, à la jonction de la peau foncée et claire.

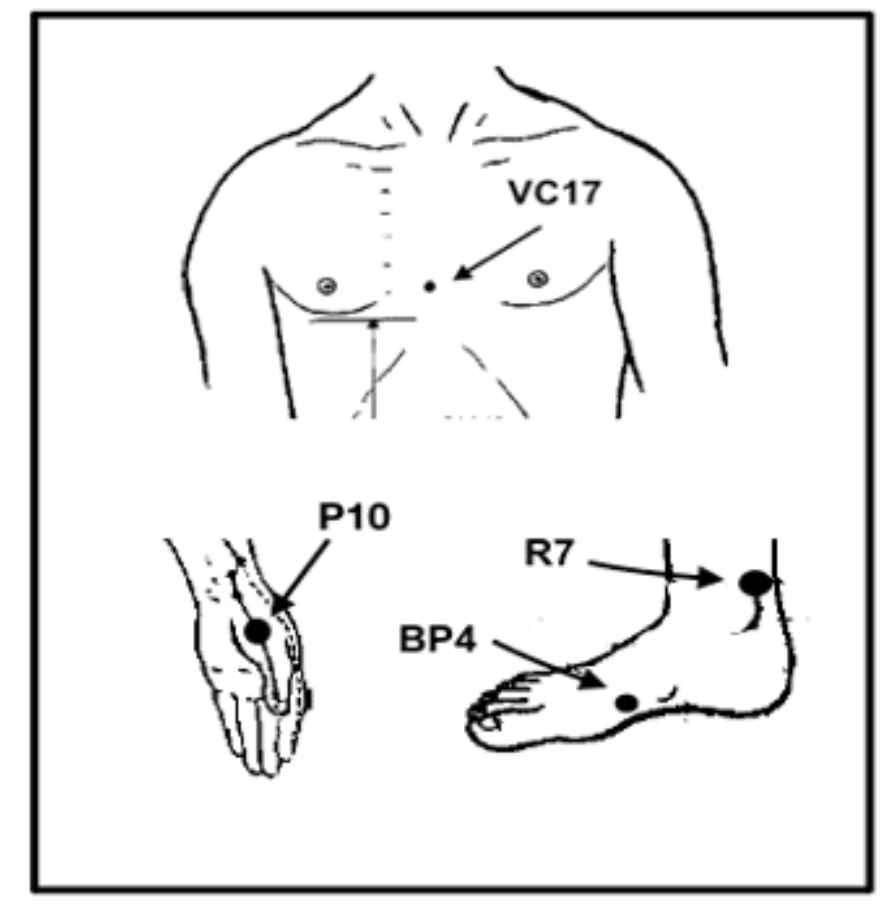

Source: Wen ${ }^{17}$ 
Fonseca ${ }^{45}$, d'autre part, a utilisé un échantillon avec 40 individus masculins pratiquant la souche musculaire deux groupes : le contrôle et l'intervention pour évaluer les effets de l'acupuncture sur l'endurance musculaire localisée (RML) des membres supérieurs. Le groupe témoin a été soumis au test RML pendant 1 minute, passant par une période de repos de 30 minutes et après, le test a été répété.

Dans le groupe d'intervention, le test a été appliqué : le test; reposé pendant 5 minutes; l'application de l'acupuncture, tonifier les points: pancréas de la rate 3 (figure $1 \mathrm{~A}$ ); rein 7 (figure 16); estomac 36 (figure 1B); vase du gouverneur 4; triple chauffe-eau 15 (figure 7); pendant 25 minutes. Après applicabilité, le test RML répété, résultant en une amélioration significative de la performance musculaire du groupe d'intervention.

En 2015, Moniz a utilisé 12 athlètes de rugby où ils ont été distribués en groupe témoin et en groupe d'essai, effectuant l'acupuncture pendant 20 minutes dans les points : coeur 5 , vessie 15, vessie 44 (figure 17) et circulation et sexualité 6 (figure 13). Lors de la deuxième séance hebdomadaire, des crevaisons ont été effectuées aux points de stress et aux points de déclenchement. Les résultats suggèrent clairement que l'application de l'acupuncture apporte des avantages aux athlètes sur les problèmes musculaires, avec une récupération totale au niveau de la douleur et la limitation des mouvements avant le jeu suivant la blessure. 
Figure 17: coeur 5 - sur le côté ventral et ulnaire de l'avant-bras, sur le côté ulnaire du tendon du muscle du fléchisseur ulnaire carpien; 1 tsun au-dessus de la poignée; vessie 15 - au niveau inférieur du bord du processus spinal de la vertèbre (T5), sur la même ligne verticale que le point Feishu (B13); vessie 44 - 3 tsun, latérale de l'axe, au niveau inférieur de bord de la colonne vertébrale des vertèbres (T6).

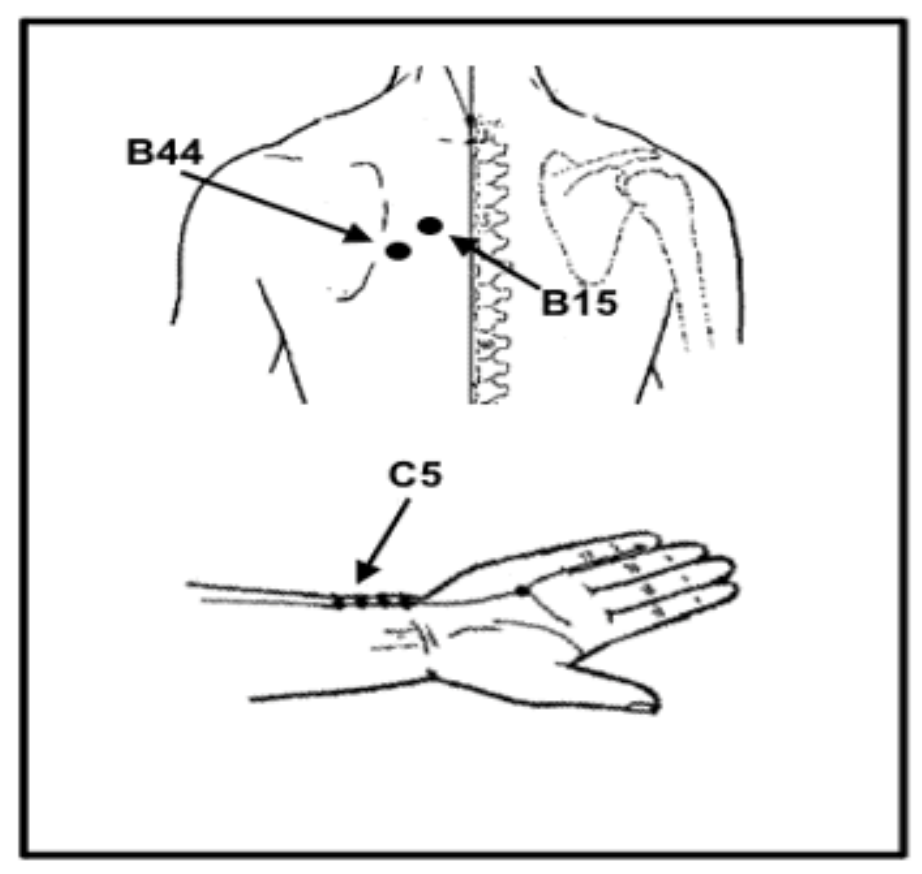

Source: Wen ${ }^{17}$

La plupart des études présentent des résultats significatifs en ce qui concerne l'amélioration de la performance physique, ce qui conduit à une amélioration dans le temps de la fin des tests. Cela provoque des personnes soumises à un traitement d'acupuncture, recevant une activation de la neurophysiologie de contrôle de la douleur, déclenchant une amélioration de la performance musculaire, influençant grandement le résultat final. ${ }^{46}$

\section{ACUPUNCTURE UTILISÉE DANS LE TRAITEMENT DES LÉSIONS}

L'acupuncture agit dans ce processus de guérison de la maladie, l'élimination de sa cause, et est donc administré à la fois pour le traitement de diverses douleurs aigues 
et chroniques. II peut être utilisé dans les interventions chirurgicales, dans l'amélioration des conditions physiques et dans la prévention des blessures dans divers sports. ${ }^{24}$

Actuellement, les athlètes et leurs entraîneurs cherchent à améliorer la performance physique et la prévention grâce à une formation exhaustive, un suivi nutritionnel, la prévention physiothérapeupique, des plans d'entraînement, le suivi psychologique et l'utilisation de nouveaux l'équipement sportif pour atteindre les objectifs. Même avec ces systèmes améliorés, de nombreux athlètes préfèrent et se déplacent sur la voie la plus rapide, en utilisant le traitement illicite, comme l'utilisation d'anabolisants et de drogues. ${ }^{32}$ Selon Frasca 35, il affirme que l'acupuncture fonctionne dans l'être humain dans son ensemble, le traitement de la douleur, l'amélioration des performances et d'agir directement dans le système psychologique des athlètes générant une augmentation de la confiance psychique du sportif.

Selon Santos et ses collaborateurs 20 , la plupart des athlètes ont eu l'expérience de ressentir des douleurs musculaires tardives, qui apparaissent dans les périodes d'entraînement, où l'intensité et le volume de travail sont plus élevés. Un autre type de douleur est causée par une blessure, en déplaçant les athlètes loin de l'entraînement et les compétitions pendant de longues périodes. ${ }^{21}$

Basé sur la neurophysiologie, l'histoanatomical et le neurochimique, CTM explique qu'en parant le point qui est situé dans les canaux des méridiens spécifiques, il facilitera le flux d'énergie dans ces structures surchargées par les modalités sportives, atteindre des objectifs analgésiques. ${ }^{25,47}$

D'autres auteurs rapportent que l'application de la technique a l'action des opioïdes endogènes, la libération de â-endorphine qui est identifiée dans la modulation de douleur et l'inhibition de la transmission nociceptive à tous les niveaux du système nerveux se produit.) par l'utilisation de l'électroacupuncture, régulation positive de l'expression du rapport de synthèse d'oxyde nitrique neural (nNOS) / diaphorase de NADPH (NADPHd) se produit. mesuré par des voies thalamic et la médulle dorsale. II 
y a également quelques données suggérant qu'en plus des opioids, d'autres mécanismes sont impliqués dans le traitement de la douleur. ${ }^{6,48,49,50,51}$

La présence de NON favorise l'amélioration de la vascularisation locale, indiquant une action homéototique de l'acupuncture, mais l'augmentation de NON dans le fascicle glacé peut clarifier cette action réglementaire des réflexes somatiques causés par la technique. ${ }^{6,52}$ D'autres études observent la libération de la sérotonine après stimulation d'aiguille. ${ }^{51,52,53}$

L'acupuncture est appliquée au tonifier, détendre les muscles des athlètes et maintenir l'équilibre énergétique du corps. En plus de l'effet analgésique, il est possible de stimuler d'autres facteurs biologiques, activant l'hypothalamus et la glande pituitaire atteignant des effets systémiques, tels que : les neuro-hormones et les neurotransmetteurs accrus. 2 améliorer la performance de l'athlète dans ses modalités respectives, ainsi contrôlées par les systèmes, nerveux, endocriniens et immunitaires. $36,54,55,56,57,58,59,60$ De nombreuses études ont montré que l'acupuncture fonctionne dans ces trois systèmes, fournissant: soulagement des tensions et des douleurs musculaires; augmentation du flux sanguin local; libération d'opioïdes endogènes, diminution de la perception de la douleur; amélioration de la modulation de l'équilibre sympathique et parasympathique du système nerveux; modulation du système immunitaire; l'activation du tissu conjonctif et des muscles libérant des myosines qui ont des effets anti-inflammatoires, libérant des substances antioxydantes, diminuant

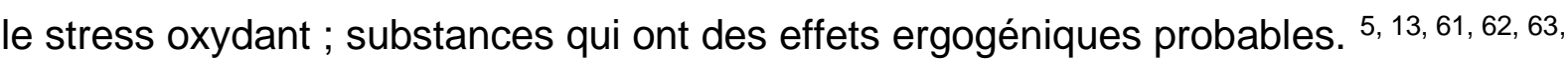
64

Dans une étude de laboratoire avec des rats, Lee et collaborateurs 65 ont rapporté que l'animal lorsqu'il a été soumis à l'acupuncture et l'exercice a présenté une suppression des effets sur 5 HT (5 hydroxytryptamines) et l'expression de THP (hydroxylase tryptophane) dans la rafe dorsal des cobayes, atteignant l'effet ergogénique, puisque le 5 HT empêche la performance de l'exercice chez l'homme et les rats. 
Dans une étude, Zyloney et 66 collabor ${ }^{\text {at }}$ eurs ont rapporté qu'il est possible de trouver une activité différente dans le cerveau, par l'évaluation de résonance magnétique, pendant la procédure d'aiguille. Ces changements apparaissent dans des régions telles que : la matière grise épiaquedrale, l'hypothalamus, le cortex somatosensoriel primaire, le gyrus temporel supérieur, le cortex cingulaire antérieur rostral et la région médiale du cortex occipital. ${ }^{48}$

Dans ses recherches, Minori ${ }^{67}$ a traité des blessures répétitives de stress à l'épaule, avec la combinaison de points de suture merveilleux de navire : triple chauffe-eau 5 vésicule biliaire 41 (figure 18) qui sont employés pour la douleur chronique et l'intestin grêle 3 (figure 3B) - vessie 62 ( Figure 11B) pour la douleur aigue.

Figure 18: triple chauffe-eau 5 - 2 tsun au-dessus du pli dorsal du poignet, entre les tendons du muscle extenseur numérique commun et le muscle du cinquième chiffre lui-même; vésicule biliaire 41 - dans la dépression entre les quatrième et cinquième métatarsiens.

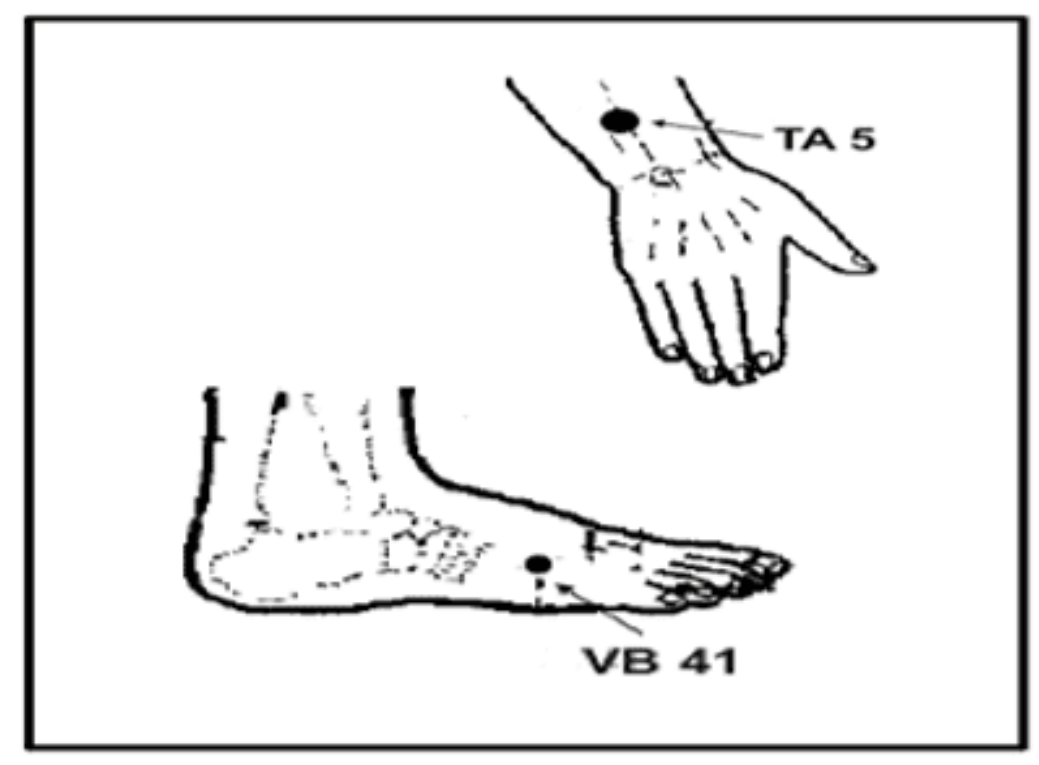

Source: Wen ${ }^{17}$

Les points locaux pour le traitement sont: gros intestin 5 pour la douleur au poignet; gros intestin 4 soulage la douleur et les oœdèmes (figure $4 \mathrm{~A}$ ); douleur au intestin grêle 
9 bras; triple chauffage 14 indiqué pour la douleur et l'inflammation de l'épaule; gros intestin 14 (figure 19) utilisé pour la raideur, la paresis, la mobilité réduite de l'épaule. Points éloignés, douleurs à l'épaule supérieures et antérieures : estomac 36 pour dissiper les facteurs pathogènes externes; estomac 37 indiqué pour la douleur, la sensibilité et les troubles de la circulation; l'estomac 41 élimine l'humidité, la stagnation, le vent et apaise la shen (esprit); l'estomac 38 élimine les douleurs aigues et les troubles articulaires de l'épaule (figure 1B). Pour les douleurs postérieures et supérieures à l'épaule : vésicule biliaire 34 indiquée pour une mobilité réduite, des spasmes, des troubles de la circulation et des troubles fonctionnels des muscles et des tendons; la vésicule biliaire 38 disperse le vent et libère de la chaleur, active le méridien et ses vaisseaux (figure 11B). Scapula de douleur, d'autre part: la vessie 65 pacifis et renforce l'esprit, libère la chaleur et soulage la douleur; la vessie 64 supprime les spasmes et détend les tendons; la vessie 60 soulage la douleur et active les points méridien et émotionnels (figure $2 \mathrm{~A}$ ); la vessie 18 favorise le calme général et élimine les spasmes (figure 19) et le foie 8 détend les tendons (figure 11A). 
Figure 19 : intestin grêle 9 - dans la région posttero-inférieure de l'articulation de l'épaule, sur le côté postteroinferior du muscle rond plus grand ; triple chauffage 14 dans la dépression entre l'achromic et le tuberculo plus grand d'humérus, au bord du tendon infraspinal ; gros intestin 14 - couvre le côté latéral et un peu radial du bras, au point distal du muscle deltoïde, 3 tsun en dessous du point jianyu; vessie 18 - sur la même ligne verticale que le geshu (B17), au niveau inférieur du bord du processus spinal de la vertèbre (T9).

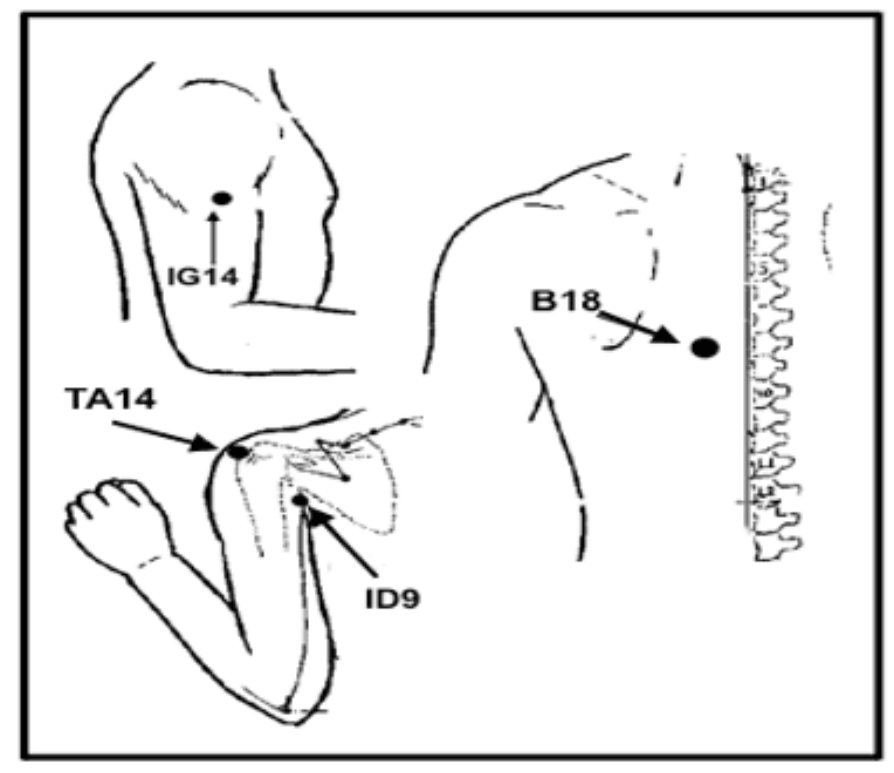

Source: Wen ${ }^{17}$

Brum ${ }^{68}$, d'autre part, associé dans son étude pour traiter le syndrome pyriforme, le massage et les techniques d'acupuncture dans les couloirs, afin d'améliorer les fonctions musculaires, la flexibilité et le soulagement des symptômes. La recherche a porté sur des athlètes des deux sexes âgés de 30 à 60 ans. La séance a porté sur le massage (Shiatsu) au début pour libérer les points de stress de la région fessier et cuisse sur le côté affecté, suivie par l'aiguille, comme suit: dans le sens des aiguilles; anti-temps; l'approfondissement; superficialisation de l'aiguille dans les régions suivantes : origine et insertion du muscle piriforme (visage pelvien du sacrum et trocanter plus grand du fémur); origine du fessier moyen (face latérale de l'os iliaque); limite supérieure du fessier minimum; limite supérieure du maximum de fessier; 
l'origine des muscles ischio-jambiers (tubés sciatiques); le muscle fémoral de biceps et la diaphysis semitendine de muscle, fini avec le massage.

Les résultats ont montré l'amélioration significative, une fois liée à l'amélioration de la douleur, de la flexibilité, de la palpation de douleur dans le muscle piriforme et à l'essai pour le syndrome pyriforme. Cela montre l'accord avec l'étude de Hongwen 69, où l'efficacité de l'acupuncture était de 97,33\% et avec la recherche de France 26 qui associait les aiguilles à la kinésiothérapie atteignant un soulagement des symptômes, la potentialisation du système neuromusculaire, un retour plus rapide aux activités sportives.

Dans son travail, Rocha ${ }^{70}$, a vérifié l'amélioration de l'activation du muscle rectus fémoral, par électromyographie, après stimulation du point d'acupuncture de l'estomac 45 (figure 20). Un total de 10 participants (5 mâles et 5 femelles) ont été sélectionnés, où ils ont effectué un exercice de 2 répétitions accroupies, où ils ont choisi l'indice d'activation musculaire le plus élevé parmi les deux mouvements par le signal électromyographique. Puis l'acupuncture a été appliquée, tonifiant le point d'estomac 45 pendant 10 minutes et puis le squat isométrique a été répété dans la phase inférieure. Selon les résultats trouvés l'acupuncture a modifié l'activité électrique du muscle de rectus fémoral pendant la phase isométrique dans la phase inférieure du squat, s'avérant efficace.

Figure 20 : estomac 45 - 0.1 tsun sur le côté du coin latéral du lit d'ongle du deuxième orteil

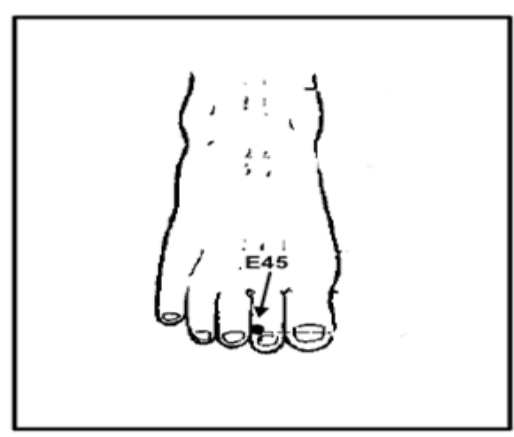

Source: Wen ${ }^{17}$

RC: 41652

Disponível em: https://www.nucleodoconhecimento.com.br/sante/acupuncture-en-prevention 
Dans une autre étude Pinheiro ${ }^{71}$, caractérisé et déposé quelques points pour le traitement de la lésion ménisque: foie 8 (figure $11 \mathrm{~A}$ ) utilisé pour tonifier le canal du foie avec la fonction de l'harmonisation et la tonalité du foie et du sang Qi, la promotion de la plénitude des reins et conduisant à la relaxation des tendons et des muscles, renforçant le Qi du genou. Le point de vessie 40 (figure 5) se trouve dans la fossa popliteal permettant : dispersion de la chaleur et vent pervers des membres; relaxation des muscles et des tendons; détruire les vaisseaux sanguins; réduit la chaleur et élimine la stase du sang; regidez diminué et renforce le genou et la lombaire. D'autre part, le point rénal 10 (figure 21) mobilise l'eau; tons yin Qi; expulse l'humidité et la chaleur perverse, traitant la douleur au genou, l'œdème de la jambe et l'engourdissement du genou et du césum popliteal.

Figure 21: rein 10 - sur le côté médial du pli popliteal, entre les muscles semi-tendineux et semi-membraous

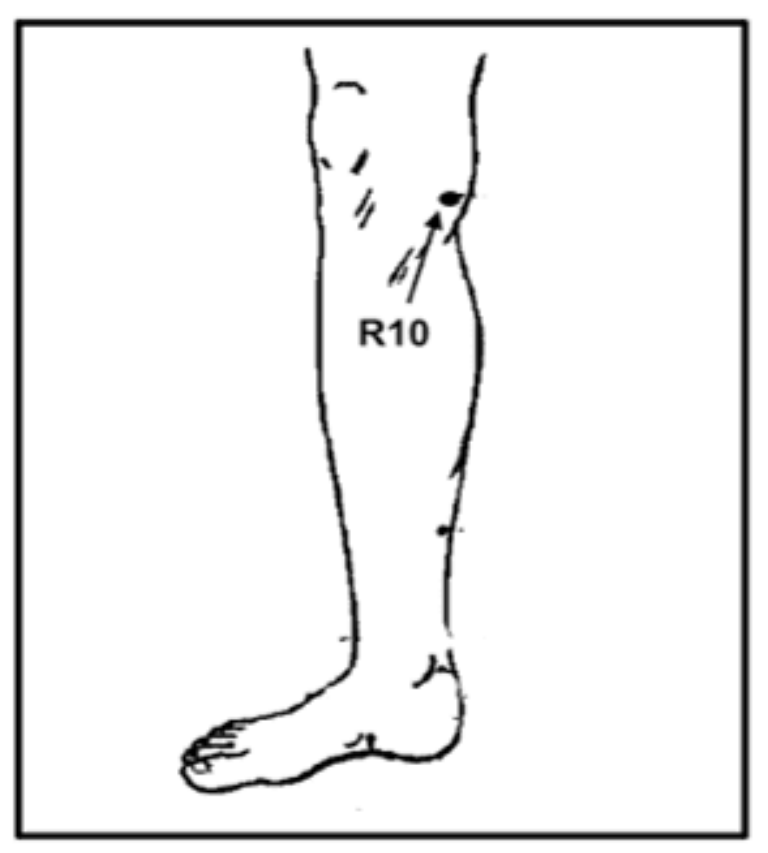

Source: Wen ${ }^{17}$

Dans une étude sur les dysfonctionnements de l'épaule, Nunes 72 , a examiné la bibliographie et a déposé les points de vaisseaux merveilleux: l'intestin grêle 3 (figure 3B) - vessie 62 (figure 11B) pour équilibrer les fonctions, la relaxation des muscles et 
des tendons; les points du triple chauffe-eau 5 - vésicule biliaire 41 (figure 18) soulagent la douleur et détendent les tendons; Points Ashi; le foie 8 (figure 11A) et le rein 7 (figure 16) pour renforcer les tendons; estomac 36 (figure 1B) éliminant le froid et l'humidité des articulations; l'intestin 4 (figure 4A) et le gros intestin 15 (figure 22) agissant dans le mouvement et la douleur de la flexion de l'épaule; triple chauffe-eau 14 (figure 19) et gros intestin 16 (figure 22) améliorant la mobilité et la douleur des extensions.

Figure 22: gros intestin 15 - sur le dessus de l'épaule, sur le bord latéral de l'acromion, il ya deux dépressions, ce point est dans la dépression antérieure; gros intestin 16 dans la dépression entre le bord supérieur et postérieur de l'articulation acromioclaviculaire et la colonne vertébrale de l'omoplate.

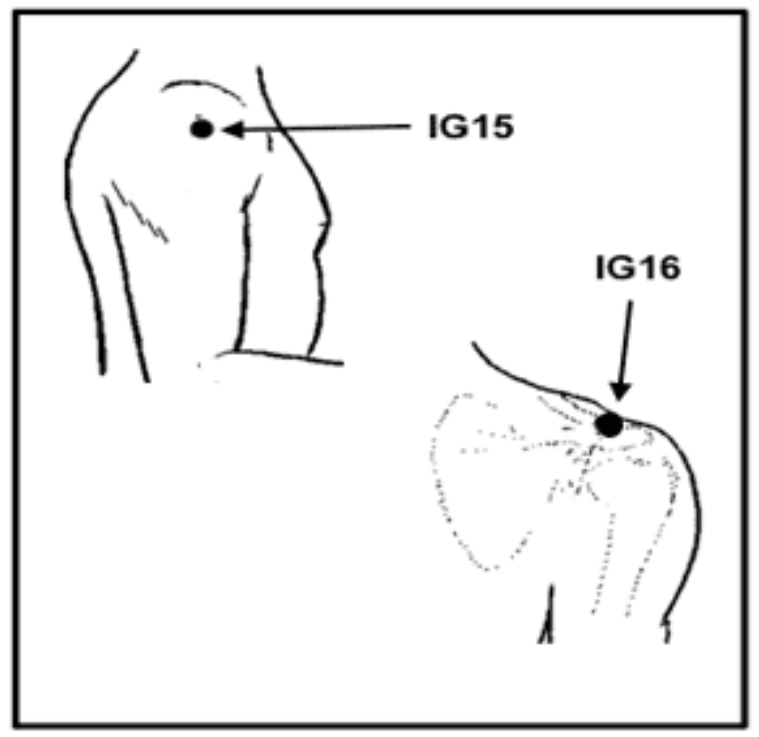

Source: Wen ${ }^{1}$

Afin de vérifier les effets immédiats de l'acupuncture sur les douleurs musculaires tardives (DMIT) et la capacité fonctionnelle contractile des muscles, Parisoto 73, a sélectionné 30 sujets ( 12 hommes et 18 femmes), âgés entre 20 et 30 ans, praticiens de l'activité 2 à 3 fois par semaine. Ils ont été divisés en trois groupes : traités avec l'acupuncture ; placebo faux; Contrôle. Les éléments suivants ont été évalués : seuil de douleur avec l'algelomètre; force musculaire isométrique maximale maximale 
maximale; activité électromyographique; perception subjective de la douleur à travers l'échelle analogique visuelle.

Après les évaluations, les volontaires ont été soumis à l'induction de DMIT, effectuant la flexion et l'extension du coude avec la charge (fil de biceps), jusqu'à la limite maximale de l'exécution de mouvement : 3 séries ont été exécutées jusqu'à épuisement de muscle avec un intervalle de 4 à 5 minutes. Après cette procédure, l'état dans lequel le muscle était avant l'acupuncture a été réévalué.

Les points choisis pour le traitement du groupe d'acupuncture étaient : gros intestin 4 (figure 4A), gros intestin 11 (figure 4B), estomac 36 (figure 1B), vésicule biliaire 34 (figure 11B), qui sont des points analgésiques et tonifiants des muscles et des tendons. Dans le groupe fictif, des points de placebo ont été employés et les aiguilles ont été introduites superficiellement ; groupe de contrôle les individus juste se poser. Les deux groupes sont restés au repos pendant 20 minutes.

Les résultats indiqués : la perception de la douleur avec l'échelle analogique a été réduite dans le groupe d'acupuncture ; le seuil de douleur par l'algelomètre a augmenté après intervention dans ce groupe. Après l'intervention d'acupuncture, l'activation de muscle de biceps a été réduite de $30 \%$ une fois liée au groupe de faux et de commande. La force musculaire, après DMIT, a diminué de $48 \%$ dans le groupe testé, étant plus faible que dans les groupes de faux et de contrôle (58\% et $56 \%$ respectivement). Tous les groupes ont présenté le déficit maximal de force de muscle isometric après le protocole d'induction de dmit : l'acupuncture a diminué de $26 \%$; imposture 29\%; contrôle 33,5\%.

Cependant, il a été conclu que l'acupuncture était efficace dans l'amélioration immédiate de la perception de la douleur et le seuil accru par algelomer, fournissant des altérations neuromusculaires, mais n'améliorant pas la performance contractile de la musculature.

Dans une autre étude de Tolentin ${ }^{74}$, il a évalué l'effet de l'auriculothérapie sur la douleur, la fonctionnalité et la mobilité des personnes avec la douleur lombaire 
chronique. Diviser 31 participants en trois groupes : expérimental avec des aiguilles de sang en acier inoxydable jetables de 1,5 mm; expérimental avec des graines de moutarde; contrôle sans intervention. L'échelle analogique visuelle a été utilisée pour évaluer la douleur; pour le test de fonctionnalité, le questionnaire sur l'incapacité lombaire du Québec et le test de la séance à la position debout ont été utilisés; la mobilité lombaire a été exécutée.

Les interventions ont été effectuées pendant 4 semaines, étant une session hebdomadaire, appliquée dans les points: Shenmen, Rim, Sympathique, Analgésie, Relaxation musculaire, Lumbar et colonne vertébrale surrénale (figure 10). Après un intervalle de 1 semaine après la dernière demande, les individus ont été réévalués avec le même protocole.

Les résultats ont montré l'amélioration de l'état de la lombalgie, ainsi que l'amélioration de la capacité fonctionnelle dans les deux groupes expérimentaux, contrairement au contrôle qu'il n'y avait pas de différence significative par rapport à la douleur initiale. Aucune différence n'a été constatée entre les techniques exécutées (aiguille et graine), ce qui indique que les deux sont bénéfiques pour le soulagement de la lombalgie chronique et l'augmentation de la capacité fonctionnelle.

Figure 23 : Points d'oreille

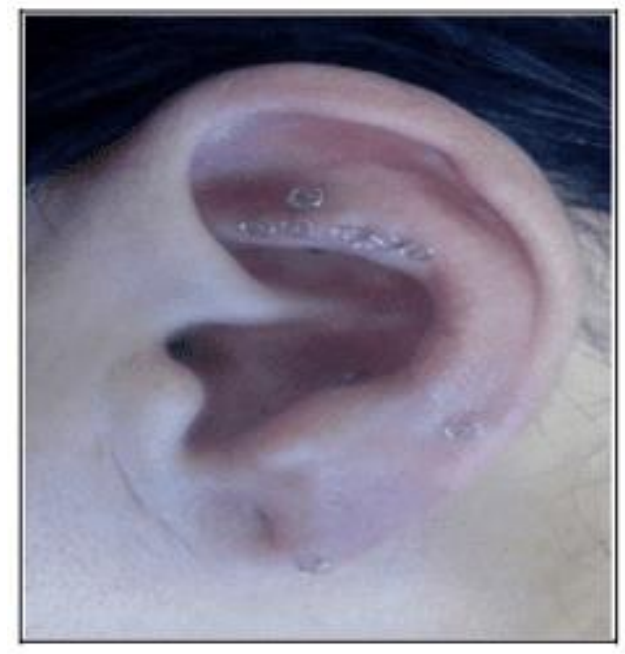

Source: Tolentino ${ }^{74}$

RC: 41652

Disponível em: https://www.nucleodoconhecimento.com.br/sante/acupuncture-en-prevention 


\section{CONSIDÉRATIONS FINALES}

Basé sur la littérature, analysant tous les résultats et leurs preuves scientifiques, il est considéré que le traitement d'acupuncture est efficace dans le traitement, l'amélioration de la performance et la prévention des blessures chez les athlètes professionnels et amateurs. Les personnes recevant l'intervention, même si elles ont un certain type de complication blessée, le problème est moins grave et rétablit rapidement la fonction organique, c'est-à-dire, l'athlète qui souffre d'un certain type de blessure et effectue l'acupuncture, cette adversité aura tendance à être minime et les activités de retour précoce.

Même les athlètes qui n'utilisent pas l'acupuncture afin de prévenir la prévention et de présenter la lésion, l'intervention de ce présente des preuves cliniques, basée sur la neurophysiologie, la libération d'opioïdes, en mettant l'accent sur le traitement de la douleur et l'amélioration du bien-être du patient .

L'intervention d'acupuncture, bien qu'il s'agit d'une technique millénaire, est toujours en croissance en Occident, a obtenu de grands résultats dans la pratique clinique, les questions de prévention des blessures musculo-squelettiques, et aussi dans la prévention et le traitement des maladies douleur psychosociale, aigue et chronique.

En raison de l'équilibre du système énergétique, l'acupuncture traite non seulement le bien-être physique, mais aussi le bien-être mental, parce que le fondement théorique de la technique ne sépare pas le corps de l'esprit bénéficiant du stress émotionnel, réduisant l'anxiété et équilibrant le l'esprit humain.

\section{RÉFÉRENCES}

1. Ching N. O livro de ouro da medicina chinesa. 3. ed. Rio de Janeiro: Objetiva; 1991.

2. Malveira CL, Souza FCS, Rocha TBX. Biomecânica do movimento isométrico de pedala antes e após aplicação de acupuntura: Um estudo preliminar. Motric. 2012 Set;8(52):630-9. 
3. Sousa NR. Relação entre a reatividade de pontos de acupuntura e atividade física com afecções ortopédicas determinadas por exames de imagens em equinos. Botucatu. Tese [Doutorado em Biotecnologia Animal] Universidade Estadual Paulista; 2015.

4. Silveira NC, Martins RCC. Eficácia da acupuntura para o tratamento da fibromialgia: Uma revisão sistemática. Psicol e Saúde em Debate. 2018 Fev; 4(1):85-105.

5. Sant' Anna FM, Rangel VM, Mota DDS. Tratamento da Tenossinovite de De Quervain por Acupuntura - Relato de Caso. Vittalle. 2018 Mar; 30(1):130-6.

6. Tsuchiya M, Sato EF, Inoue M, Asada A. Acupuncture enhances generation of nitric oxide and increases local circulation. Anesth Analg. 2007 Feb; 104(2):3017.

7. Tavares MG, Machado AP, Motta BG, Borsatto MC, Rosa AL, Xavier SP. Electro-acupuncture efficacy on pain control after mandibular third molar sugery. Brz Dent J. 2007; 18(2):158-62

8. Hutchinson AJ, Ball S, Andrews JC, Jones GG. The effectiveness of acupuncture in theating chronic non-specific low back pain: a systematic review of the literature. J Otthop Surg Res. 2012 Oct 30;7:36.

9. Moniz PJL. Avaliação da acupuntura no desempenho do rugby. Coimbra. Dissertação [Mestrado Integrado em Medicina] - Faculdade de Medicina da Universidade de Coimbra; 2015.

10. Magalhães PC. Efeito da acupuntura na prevenção da hemorragia pulmonar induzida por exercício em cavalos puro sangue inglês de corrida. Botucatu. Dissertação [Mestrado em Biotecnologia Animal] - Universidade Estadual Paulista; 2016.

11. Matias $B$ et al. Acupuntura de equilíbrio como coadjuvante no tratamento de disfunções temporomandibulares. Ação Odonto. 2018 Jun; 1(1):65-72.

12. Franco A. Acupuntura Ajuda Atletas nas Lesões e na Performance. Jornal da Madeira 2016 agosto 2;p.25.

13. Lopes SS, Mota MPG. Efeito da acupuntura na resistência física após exercícios repetitivos de tornozelo - Estudo experimental. R bras Ci e Mov. 2018 Jul;26(1):13-21. 
14. Lopes SS, Mota MPG. Influência da acupuntura no limiar de percepção dolorosa de musculatura submetida a esforço repetitivo. Br J Pain. 2018 Jul; 1(3):207-11.

15. Cohen M, Abdala RJ, Ejnisman B, Amaro JT. Lesões Ortopédicas no futebol, Rev. Bras. Ortop 1997 Dez;32(12):940-44.

16. Dias Junior JC, Marino DM. Acupuntura na prevenção de lesões musculares em atletas de futebol profissional. Rev Fisioter S Fun. Jan-Jul:6(1):36-42.

17. Wen TS. Acupuntura Clássica Chinesa. 15. ed. São Paulo: Cultrix, 2006.

18. Maciocia G. Os fundamentos da medicina chinesa: um texto abrangente para acupunturistas e fitoterapeutas. São Paulo: Roca; 1996.

19. Ross J. Combinações dos Pontos de Acupuntura: A Chave para o Êxito Clínico. 1 ed. São Paulo: Roca, 2003.

20. Santos VC, Kawano MM, Banja RA. Acupuntura na melhora da performance em atletas juvenis de handebol, Rev Saúde e Pesq 2008 Set-Dez;1(3):331-35.

21. Rubio K, Godoy Moreira F. A dor em corredores com fascite plantar: o uso da acupuntura, Rev Dor 2008 Jul-Set;9(3):1290-1296.

22. Pires TF, Pellegrinotti IL. Acupuntura na Performance Atlética: Estudo Exploratório. [8 $8^{\text {a }}$ Mostra Acadêmica da UNIMEP; 2010 out; 1-4; Piracicaba, Brasil].

23. Yang HY, Liu TY, Gao M. Electrical acupoint stimulation increases athletes rapid strength, Zhongguo Zhen Jiu 2006 May;26(5): 313-315.

24. Luna MP, Fernandes Filho J. Efeitos da Acupuntura na performance de Atletas velocista de alto rendimento do Rio de Janeiro. Fit e Perform J 2005 Julh/Ago;4(4):199-214.

25. Ehrlich D, Haber P. Influence of acupuncture on pysical performance capacity and Haemodynamic Parameters. J. Sports Med 1992 May;13(6): 486-91.

26. França D, Fernandes-Senna V, Cortez CM. Acupuntura cinética como efeito potencializador dos elementos moduladores do movimento no tratamento de lesões desportivas. Fisioter Bras 2004 mar-abr; 5(2):111-8.

27. Wadsworth L T. Acupuncture in sports medicine. Curr Sports Med Rep 2006 Feb; 5(1):1-3. 
28. Barela J A. Estratégias de Controle em Movimentos Complexos: Ciclo Percepção - Ação no Controle Postural. Rev Paul de Educ Fís 2000; 3: 79-88.

29. Papler PG et al. Reabilitação do joelho. In: Greve J.M. A. e Amattuzzi, M.M. Medicina de reabilitação aplicada à ortopedia e traumatologia. São Paulo: Roca, 1999.

30. Gemeo LH. Ignatti C. Acupuntura como Ferramenta Auxiliar do Aumento da Performance Desportiva. In: Anais do Simpósio Internacional de Ciências Integradas da UNAERP; 2004 ; Guarujá, Brasil. São Paulo: Universidade de Ribeirão Preto Campus Guarujá; 2004. p. 1-9.

31. Rossetto SC. Acupuntura nos Esportes. 1 ed. São Paulo: Phorte, 2009.

32. Pelham TW, Holt LE, Stalker R. Acupuncture in human Performance. J Strength Cond Res 2001 May;15(2):266-71.

33. Myamoto T. Acupuncture treatment for muscle injury. Japa Journ of Phys Fit and Spor Med 1997; 43(2):39-41.

34. Fry AC, Kraemer KJ. Resistance exercise overtraining and overreaching: neuroendocrine responses. Sports Med 1997 Feb;23(2): 106-29.

35. Frasca L. Desempenho na Ponta da Agulha. Rev Farmac 2011 out-nov:44-5.

36. Costa V. Acupuntura Previne Lesões e ajuda a melhorar o Desempenho nas Pistas [periódico na internet]. 2013 [acesso em 22 jan 2017] Disponível em: http://blogs.oglobo.globo.com/pulso/post/acupuntura-previne-lesoes-ajudamelhorar-desempenho-nas-pistas-500798.html.

37. Luna M. Os Benefícios da Acupuntura no Esporte. 2016 [acesso em 22 jan 2017] Disponível em: http://www.ibramrp.com.br/noticia/49/os-beneficios-daacupuntura-no-esporte.

38. Akimoto T, Nakahori C, Aizawa K, Kimura F, Fukubayashi T, Kono I. Acupuncture and responses of imunoligic and endocrine markers during competition, Med Sci in Sports Exerc 2003 Ago;35(8):1296-1302.

39. Bopp-Limoge C. L’acupuncture Permet-elle D’ameliorer les Performances Sportives Stude Personnelle a Propos de 35 Sportifs de haut niveau. These Medicine 1998; 117:215. 
40. Lin ZP et al. Effects of acupuncture stimulation on recovery ability for male elite basketball athletes. Amer Jour of Chin Med 2009; 37(3):471-81.

41. Lin, ZP et al. Effect of auricular acupuncture on oxygen consumption of boxing athletes. Chin Med Jour 2009; 22(13):1587-90.

42. Dhillon $S$ et al. The acute effect of acupuncture on $20-\mathrm{km}$ cycling performance. Clin Jour of Spo Med 2008; 18(1):76-80.

43. Hubscher $\mathrm{M}$ et al. Immediate effects of acupuncture on strength performance: a ramdomized, controlled crossover trial. Euro Jour of Appli Physi 2010; 110(2):353-58.

44. Geng $L \mathrm{~J}$ et al. Investigation on the effects of ear acupressure on exerciseinduced lactic acid levels and the implications for athletic training. Amer Jour of Acupu 1995; 23(4).

45. Fonseca LP, Lessa JFM. Efeito da Aplicação da Acupuntura na Resistência Muscular Localizada de Membros Superiores em Praticantes de Exercício Resistido. Brasília. Monografia [Graduação em Fisioterapia] - Centro Universitário de Brasília; 2011.

46. Belmiro H, Vicentini D, Camilotti CM. Efeitos da Acupuntura no Desempenho Motor de Atletas. Rev Fac Educ Fis 2013 jul-set; 11(3):176-91.

47. Angeli AL, Fernandes JGJ, Luna SPL. Acupuncture Applied Equine Sports Medicine, Ver Acad 2007 Jul-Set;5(3):325-33.

48. Staud R. Mechanisms of acupunture analgesia: effective therapy for musculoskeletal pain? Curr rheumatol Rep. 2007 Dec;9(6):473-81

49. Bucinskaite V, Lundeberg T, Stenfors C, Ekblom A, Dahlin L, Theodorsson E. Effects of electro-acupuncture and pysical exercice on regional concentrations of neuropeptides in rat brain, Brain research 1994 Dec;666(1): 128-32.

50. Shang C. Prospective tests on biological models of acupuncture. Evid Based Complement Alternat Med. 2009 Mar; 6(1):31-9.

51. Bowsher D. Mechanisms of acupuncture. In: Filshie J, White A. editors. Medial Acupuncture: a western scientific approach. London: Churchill Livingstone; 1988:69-82.

52. Ma SX. Neurobiology of acupuncture: Toward CAM. Evid Based Complement Alternat Med. 2004 Jun;1(1):41-7. 
53. Li J, Wang Q, Liang H, Dong H, Li Y, Ng EH, et al. Biophysical characteristics of meridians and acupoints: a systematic review. Evid Based Complement Alternat Med. 2012;2012793841.

54. Cardeal EL, Oliveira KB, Aoki MN, Amarante MK, Oliveira CEC, Suzuki S, et al. Aspectos imunológicos da acupuntura. Biosaúde 2005 Dez:7(1/2):49-60.

55. Szabó MVRS, Bechara GH. Acupuntura: Bases Científicas e Aplicações. Ciência rural 2001:31(6):1091-99

56. Saad M. A Medicina tradicional chinesa tem base científica?. Einstein: Educ Contin Saúde 2008: 6(3):122-6.

57. Alvarenga TF, Amaral CG, Steffen CP. Ação da acupuntura na neurofisiologia da dor: revisão bibliográfica. Rev Amazôn Scienc \& Helth 2014 Out/Dez:2(4):29-36.

58. Pereira FAO. Evidências científicas da ação da acupuntura. Perspectivas 2005 jan/jul:4(7):88-105.

59. Taffarel MO, Freitas PMC. Acupuntura e analgesia: aplicações clínicas e principais acupontos. Ciênc Rural 2009 Dez:39(9): 2665-72.

60. Lopes LF, Lopes MC, Fialho FAP, Gonçalves AL. Sistema de conhecimento para diagnóstico em acupuntura:uma modelagem usando o CommonKADS. Gest Prod 2010 Dez:x(x):1-15.

61.Zhu J, Arsovska B, Kozovska K. Acupuncture treatment for sports injury hamstring muscles group, Inter J Clin Exper Med Sci 2017 Nov;3(6):71-3.

62. Vickers AJ, Cronin AM, Maschino AC, Lewith G, MacPherson H, Foster NE, et al. Acupuncture for chronic pain individual patient data meta-analysis, Arch Intern Med 2012 Oct;172(19):1444-53.

63. Toda S. Effect of acupuncture on carnitine for skeletal muscle fatigue, Chin Med 2012 Jan;3:9-12.

64. Lopes SS, Mota MPG. Influência da acupuntura no limiar de percepção dolorosa de musculatura submetida a esforço repetitivo, Br J Pain 2018 julset;1(3):207-11.

65. Lee, SH, Chung, SH, Lee, JS, Kim, SS, Shin, HD, Lim, BV, et al. Effects Acupunturaq on the 5-hydroxytryptamine synthesis and tryptophan hydroxylase 
expression in dorsal in the dorsal raphe of exercice rats. Neurocienses Letters 2002 Oct;332(1): 17-20.

66. Zyloney CE, Jensen K, Polich G, Loiotile RE, Cheetham A, LaViolette PS, et al. Imaging the functional connectivity of the Periaqueductal Gray during genuine and sham electroacupuncture treatment. Mol pain. 2010 Nov;6:80

67. Minori AET, Mejia DPM. Atuação da Acupuntura para o Tratamento de LER/DORT no Ombro. Goiânia. Monografia [Pós Graduação em Acupuntura] Faculdade Ávila; 2007.

68. Brum KN, Alonso, AC, Brech GC. Tratamento de massagem e acupuntura em corredoresrecreacionais com síndrome do piriforme. Arq Cienc Sau 2009 abrjun; 16(2): 62-6.

69. Hongwen S. Clinical Observation on Acupuncture Treatment of Piriformis Syndrome. J Tradit Chin Med. 2003 mar; 23(1):38-9.

70. Rocha TBX, Vilela Junior GB, Martins GC, Manzatto L, Grande AJ. Análise Comparativa Eletromiográfica do Reto Femoral em Isometria na Posição Inferior do Agachamento Wall Slide, antes e após a Aplicação da Acupuntura no Ponto ST45. Rev Bras Cien e Mov 2012 jul; 20(4):92-8.

71. Pinheiro RG, Mejia DPM. Efeito da Acupuntura na Melhora do Paciente com Quadro Álgico de Lesão de Menisco Medial. Goiânia. Monografia [Pós Graduação em Acupuntura] - Faculdade Ávila; 2012.

72. Nunes EA, Mejia DPM. Tratamento de Acupuntura para Combater Dores nos Ombros. Goiânia. Monografia [Pós Graduação em Acupuntura] - Faculdade Ávila; 2012.

73. Parisotto D. Efeito Imediato da Aplicação da Acupuntura na Dor Muscular Tardia e na Capacidade de Contração Muscular. Curitiba. Dissertação [Mestrado em Fisiologia] - Universidade Federal do Paraná; 2014.

74. Tolentino F. Efeito de um Tratamento comAuriculoterapia na Dor, Funcionalidade e Mobilidade de Adultos com Dor Lombar Crônica. Rio Claro. Dissertação [Mestrado em Desenvolvimento Humano e Tecnologias] Universidade Estadual Paulista - UNESP; 2016.

Soumis: Avril, 2019. 
Approuvé : octobre 2019. 